\title{
Composição taxonômica e distribuição de anuros no Alto e Médio Paranapanema, estado de São Paulo
}

\author{
Cybele de Oliveira Araujo ${ }^{1,4}$, Ciro Koiti Matsukuma ${ }^{2}$ \& Selma Maria de Almeida Santos ${ }^{3}$ \\ ${ }^{1}$ Seção de Animais Silvestres, Instituto Florestal, Rua do Horto, 931, \\ Horto Florestal, CEP 02377-000, São Paulo, SP, Brasil \\ ${ }^{2}$ Seção de Manejo e Inventário Florestal, Instituto Florestal, Rua do Horto, 931, \\ Horto Florestal, CEP 02377-000, São Paulo, SP, Brasil \\ ${ }^{3}$ Laboratório de Ecologia e Evolução, Instituto Butantan, Av. Dr. Vital Brazil, 1500, \\ CEP 05503-900, São Paulo, SP, Brasil \\ ${ }^{4}$ Autor para correspondencia: Cybele de Oliveira Araujo,e-mail: cyaraujo@if.sp.gov.br
}

ARAUJO, C.O., MATSUKUMA, C.K. \& AMEIDA-SANTOS, S.M. Taxonomic composition and distribution of anurans in the Upper and Middle Paranapanema, São Paulo state, Brazil. Biota Neotrop.13(3): http:// www.biotaneotropica.org.br/v13n3/en/abstract?inventory+bn01713032013

\begin{abstract}
The anurans of São Paulo state are the best known in Brazil. However, there are still considerable gaps in the sampling of anurans in the state, especially within the natural vegetation remnants of the Paranapanema River watersheds. The purpose of this study was to collect information on the taxonomic composition, distribution, and conservation status of anurans in the Upper and Middle Paranapanema, thus helping to identify the municipalities poorly known for this taxon in these regions. Of the 91 municipalities analyzed, only $15(16 \%)$ could be classified as relatively well sampled, having been studied for long periods by various researchers. We recorded 124 anuran species belonging to 36 genera and 12 families (Brachycephalidae, Bufonidae, Centrolenidae, Ceratophryidae, Craugastoridae, Cycloramphidae, Odontophrynidae, Hemiphractidae, Hylidae, Hylodidae, Leptodactylidae, and Microhylidae). The species richness of the anurans found in the Upper Paranapanema was far greater than that in the Middle Paranapanema. The species richness gradually decreases from the southern and southeastern portion of the Upper Paranapanema, where ombrophilous forest predominate, towards the Middle Paranapanema, where Cerrado formations and seasonal semideciduous forest are dominant. The findings of this study are important to expanding our knowledge on anuran diversity in the Cerrado and Atlantic Forest, which are two priority global hotspots for biodiversity conservation in Brazil.
\end{abstract}

Keywords: amphibians, Atlantic Forest, Cerrado, conservation, inventory, Paranapanema River watersheds.

ARAUJO, C.O., MATSUKUMA, C.K. \& AMEIDA-SANTOS, S.M. Composição taxonômica e distribuição de anuros no Alto e Médio Paranapanema, estado de São Paulo. Biota Neotrop. 13(3): http://www.biotaneotropica. org.br/v13n3/pt/abstract?inventory+bn01713032013

Resumo: A fauna de anuros do estado de São Paulo pode ser considerada a mais conhecida no país. No entanto, ainda existem consideráveis lacunas amostrais no estado, sobretudo em remanescentes de vegetação natural das bacias hidrográficas do rio Paranapanema. Neste sentido, o objetivo deste estudo foi reunir informações sobre a composição taxonômica, distribuição e o status de conservação dos anuros no Alto e Médio Paranapanema, auxiliando na identificação dos municípios que ainda permanecem como vazios amostrais deste táxon nestas bacias hidrográficas. Dos 91 municípios avaliados, apenas 15 (16\%) podem ser classificados como relativamente bem amostrados, tendo sido estudados por longos períodos e vários pesquisadores. Registrou-se um total de 124 espécies de anuros, pertencentes a 36 gêneros e 12 famílias (Brachycephalidae, Bufonidae, Centrolenidae, Ceratophryidae, Craugastoridae, Cycloramphidae, Odontophrynidae, Hemiphractidae, Hylidae, Hylodidae, Leptodactylidae e Microhylidae). A riqueza de anuros registrada no Alto Paranapanema é muito superior à encontrada no Médio Paranapanema, existindo uma diminuição gradual na riqueza de espécies partindo da porção sul e sudeste do Alto Paranapanema, onde predominam a floresta ombrófila, em direção ao Médio Paranapanema que apresenta uma dominância das formações de Cerrado e floresta estacional semidecidual. Este estudo é uma importante contribuição para ampliar o conhecimento sobre a diversidade de anuros no Cerrado e Mata Atlântica, os dois hotspots mundiais prioritários para a conservação biológica existentes no país.

Palavras-chave: anfíbios, Mata Atlântica, Cerrado, conservação, inventário, bacias hidrográficas do rio Paranapanema. 


\section{Introdução}

A fauna de anuros do estado de São Paulo pode ser considerada como a mais conhecida do país, no entanto ainda existem consideráveis lacunas amostrais no nordeste e sudeste do estado como nas bacias hidrográficas do rio Paranapanema (Rossa-Feres et al. 2011). Contudo, nos últimos anos houve um aumento significativo no número de trabalhos publicados que abordam a composição de espécies de anuros em localidades presentes nas bacias hidrográficas do Alto e Médio Paranapanema (Condez et al. 2009, Araujo et al. 2010, 2013, Brassaloti et al. 2010, Forlani et al. 2010, Araujo \& Almeida-Santos 2011, 2013, Maffei et al. 2011a,b). Até o momento, são conhecidas no estado 230 espécies de anuros (Rossa-Feres et al. 2011), o que representa $25 \%$ da riqueza de espécies encontradas no país (Segalla et al. 2012). A anurofauna do estado pode ser dividida em dois grupos. Um deles é composto pelas espécies presentes nas fitofisionomias de floresta ombrófila da encosta litorânea e serras do Mar, Mantiqueira e Bocaina e o segundo grupo por espécies associadas às formações de Cerrado e floresta estacional que ocorrem predominantemente no Planalto Ocidental Paulista (Rossa-Feres et al. 2008). O Alto e Médio Paranapanema apresentam remanescentes de Cerrado, floresta estacional semidecidual e floresta ombrófila (Kronka et al. 2005).

O Cerrado está distribuído por planaltos, depressões e planícies dominadas por formações campestres e savânicas e em menor proporção matas de galeria e floresta estacional (Oliveira-Filho \& Ratter 2002, Silva \& Bates 2002, Ab'Sáber 2005, Ribeiro \& Walter 2008). Esta heterogeneidade ambiental apresenta-se refletida em sua biota que é reconhecida mundialmente como uma das mais ricas em espécies e endemismos e encontra-se severamente ameaçada pelas atividades humanas (Colli et al. 2002, Mittermeier et al. 2004, Klink \& Machado 2005, Diniz-Filho et al. 2008). Este domínio fitogeográfico encontra-se bastante impactado, sendo a agricultura e a pecuária extensiva responsáveis pela maior parte da supressão de sua cobertura vegetal, que originalmente correspondia a 23\% do território nacional (Ratter et al. 1997, Klink \& Machado 2005, Sano et al. 2010). Aproximadamente $40 \%$ de sua vegetação natural foram devastadas e a porção mais ao sul deste domínio é a mais severamente afetada, apresentando apenas 15\% de sua extenção original (Sano et al. 2010). É justamente nas regiões sul e sudoeste do Cerrado que a riqueza de espécies de anuros tende a ser mais elevada (Diniz-Filho et al. 2008, Valdujo 2011). No estado, o Cerrado apresenta-se sob a forma de manchas dispersas, especialmente na Depressão Periférica e no Planalto Ocidental Paulista, sendo que somente $0,5 \%$ de sua cobertura vegetal encontra-se protegida por unidades de conservação que ainda sofrem ameaças como o isolamento, risco de incêndios e invasão por espécies vegetais exóticas (Durigan et al. 2004a, b, Durigan et al. 2007). Um estudo recente registrou 209 espécies de anuros no Cerrado, das quais $51,7 \%$ são endêmicas deste domínio (Valdujo et al. 2012). Dentre suas diversas formações vegetais, as fisionomias campestres e savânicas apresentam maior diversidade de espécies de anuros quando comparadas às formações florestais (Brandão \& Araújo 2002, Brasileiro et al. 2005, Valdujo et al. 2011), sendo justamente estas formações abertas as mais susceptíveis ao desaparecimento no estado (Durigan et al. 2003).

Assim como no Cerrado, processos históricos de uso e ocupação do solo levaram à supressão e fragmentação da cobertura vegetal na Mata Atlântica, restando menos de $12 \%$ de sua extensão original, o que correspondia a $15 \%$ do território brasileiro (Morellato \& Haddad 2000, Ribeiro et al. 2009). Estimativas recentes apontam que este domínio fitogeográfico apresenta-se sob a forma de fragmentos descontínuos, sendo a maior parte (80\%) menor que 50 ha (Ribeiro et al. 2009). Dentre as formações florestais brasileiras, a floresta estacional semidecidual é uma das mais ameaçadas e a que sofreu maior desmatamento, sobretudo em regiões onde a topografia propicia a agropecuária (Leitão-Filho 1987, Durigan et al. 2000, Ribeiro et al. 2009). Alguns autores consideram que sua composição florística é uma transição entre a floresta ombrófila atlântica e o Cerrado, sendo classificada como um subconjunto da flora muito mais diversa da floresta ombrófila e provavelmente, composta por espécies capazes de sobreviver a uma estação seca mais prolongada (LeitãoFilho 1987, Oliveira-Filho \& Fontes 2000, Oliveira 2006). Entretanto, estudos sugerem que esta formação vegetal seja considerada uma unidade fitogeográfica distinta, possuindo um número considerável de táxons endêmicos de plantas, lagartos e anuros (Prado 2000, Werneck \& Colli 2006, Iop et al. 2011, Werneck et al. 2011). A Mata Atlântica é o domínio brasileiro que possui a maior riqueza de anuros, apresentando mais de 400 espécies e pelo menos $85 \%$ de endemismo (Cruz \& Feio 2007), desconsiderando-se várias espécies descritas mais recentemente. Adicionalmente, 27 dos 39 modos reprodutivos de anuros conhecidos no mundo são encontrados neste domínio (Haddad \& Prado 2005). Uma vez que os modos reprodutivos são filogeneticamente conservados em anuros, é possível concluir que a diversidade filogenética desta taxocenose é maior em localidades de Mata Atlântica, que apresenta uma maior variedade de modos reprodutivos em relação aos outros domínios fitogeográficos do país (Silva et al. 2012). Considerando que a precipitação anual (umidade) é a variável climática que mais prediz estes parâmetros (Vasconcelos et al. 2010, Silva et al. 2012), espera-se que a riqueza de espécies, o número de modos reprodutivos e a diversidade filogenética não estejam distribuídos homogeneamente ao longo da Mata Atlântica. No estado, é possível identificar uma diminuição gradual na riqueza de anuros partindo das fisionomias de floresta ombrófila presentes no litoral (211 espécies) em direção ao interior, onde predominam as formações de Cerrado e floresta estacional (58 espécies) e a sazonoalidade climática é mais acentuada (Araújo et al. 2009b, Rossa-Feres et al. 2011).

Considerando que estudos realizados no Cerrado e Mata Atlântica prevêem em um cenário de mudanças climáticas alterações na disponibilidade de áreas adequadas para ocorrência de muitas espécies de anuros, em especial as endêmicas e especialistas no uso do hábitat (Haddad et al. 2008a, Silvano 2011), torna-se imprescindível a realização de inventários e estudos de monitoramento destas espécies em localidades que se mantêm como lacunas amostrais (Verdade et al. 2012). Neste contexto, o objetivo deste estudo foi reunir e sintetizar informações sobre a composição taxonômica, distribuição e o status de conservação dos anuros no Alto e Médio Paranapanema, auxiliando na identificação dos municípios que ainda permanecem como vazios amostrais desta taxocenose.

\section{Material e Métodos}

\section{Local de estudo}

As bacias hidrográficas do Alto e Médio Paranapanema referemse à porção paulista do rio Paranapanema. O Alto Paranapanema ocupa uma área de aproximadamente 2,3 milhões de hectares e apresenta em seus limites físicos 55 municípios (Kronka et al. 2005) (Figura 1). O Médio Paranapanema ocupa uma área aproximada de 1,7 milhões de hectares, abrangendo 57 municípios (Kronka et al. 2005) (Figura 1). Em sua maior parte, a bacia hidrográfica do Alto Paranapanema situa-se na Depressão Periférica Paulista, no subcompartimento denominado Depressão do Paranapanema. Esta região apresenta uma densa rede de drenagem, um relevo mais acidentado e colinas amplas mais restritas (Instituto... 1981). A porção sul e sudeste desta bacia hidrográfica está inserida no Planalto 


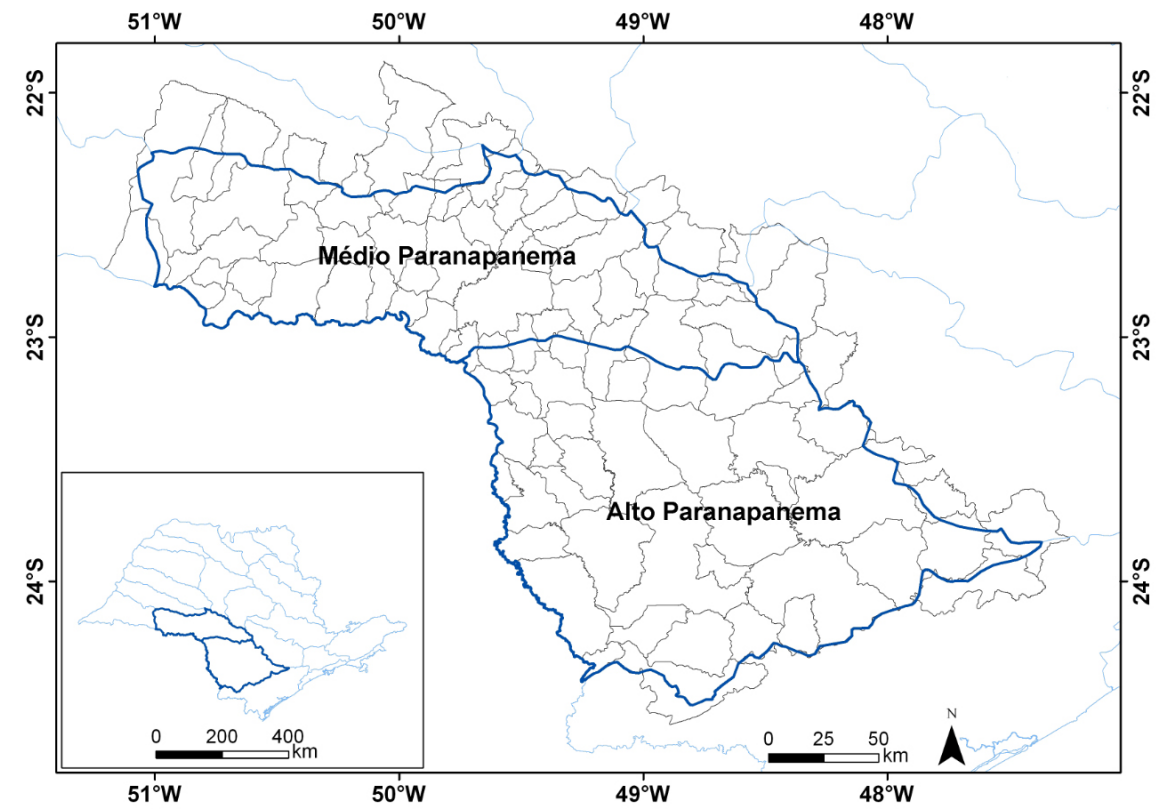

Figura 1. Municípios do Alto e Médio Paranapanema. No detalhe à esquerda estão representadas as bacias hidrográficas do estado de São Paulo, com destaque para o Alto e Médio Paranapanema.

Figure 1. Municipalities of Upper and Middle Paranapanema. On the left, note the watersheds of the São Paulo state, Brazil, highlighting the Upper and Middle Paranapanema.

Atlântico e a região mais ao norte localiza-se nas Cuestas Basálticas. Já a bacia hidrográfica do Médio Paranapanema está quase que totalmente inserida no Planalto Ocidental Paulista, apresentando um relevo levemente ondulado com predomínio de colinas amplas e baixas com topos aplainados. A drenagem é de baixa densidade com vales abertos e planícies aluviais (Ross \& Moroz 1997). Estas bacias hidrográficas se encontram em zona de transição dos tipos climáticos de Köppen: Cwa (verão quente e inverno seco) e Cfa (verão quente sem estação seca) (Setzer 1966). Nas regiões sul e sudeste do Alto Paranapanema também está presente o tipo climático $\mathrm{Cfb}$ de Köppen, caracterizado por verões amenos e ausência de estação seca (Peel et al. 2007), sendo que os maiores índices pluviométricos (entre 1.500 e $2.000 \mathrm{~mm}$ ) são encontrados nestas regiões (Lepsch et al. 1990). Nas áreas sob a influência dos tipos climáticos Cwa e Cfa, a precipitação pluviométrica anual média varia em torno de $1.425 \mathrm{~mm}$ (Durigan 2010). A cobertura vegetal é composta por remanescentes de Cerrado, floresta estacional semidecidual, floresta ombrófila densa e floresta ombrófila mista (sensu Veloso et al. 1991), estando estas duas últimas fitofisionomias presentes predominantemente na porção sul e sudeste do Alto Paranapanema (Kronka et al. 2005) (Figura 2). A vegetação natural remanescente do Alto e Médio Paranapanema corresponde a $15 \%$ e $6,4 \%$ da superfície destas bacias, respectivamente (Kronka et al. 2005).

\section{Coleta e análise dos dados}

Do total de 102 municípios presentes no Alto e Médio Paranapanema (Kronka et al. 2005) (Figura 1), foram incluídos nas análises apenas os municípios integral ou parcialmente inseridos nestas bacias hidrográficas (Figura 2). Desta forma, desconsideraramse os municípios que apenas partilham fronteiras geográficas com estas bacias como Alambari, Borã, Barra do Chapéu, Eldorado, Iporanga, Martinópolis, Porangaba, Quadra, Sete Barras, Tatuí e Torre de Pedra.
Os registros de anuros provenientes dos 91 municípios selecionados foram obtidos nas principais coleções biológicas do estado de São Paulo: Coleção de Anfíbios do Instituto de Biociências da Universidade Estadual Paulista, Rio Claro (CFBH), Coleção de Anfíbios do Instituto de Biociências da Universidade Estadual Paulista, São José do Rio Preto (DZSJRP), Coleção Herpetológica do Museu de Zoologia da Universidade de São Paulo (MZUSP) e Coleção Herpetológica do Museu de Zoologia da Universidade Estadual de Campinas (ZUEC). A identificação dos espécimes foi conferida e apenas as espécies taxonomicamente determinadas ou definidas como "cf." (confer) foram incorporadas à listagem de anuros. Exceções são a espécie Adenomera sp., coletada nos municípios de Angatuba e Guareí (Araujo \& Almeida-Santos 2013) e que pode representar uma espécie ainda não descrita (B. V. M. Berneck; A. Fouquet, comunicação pessoal) e Paratelmatobius sp. (aff. cardosoi), capturada mais recentemente no município de Apiaí (Araujo et al. 2010) e identificada como espécie em processo de descrição (C. F. B. Haddad, comunicação pessoal). Apesar da imprecisão taxonômica de Crossodactylus sp. (gr. gaudichaudii), a espécie foi adicionada à listagem por ser distinta da outra espécie do mesmo gênero registrada para as bacias hidrográficas estudadas (B. V. S. Pimenta, comunicação pessoal). Já os exemplares denominados Scinax cf. similis e Scinax $x$-signatus (aff. similis) podem tratar-se da mesma espécie (L. F. Toledo, comunicação pessoal), que é comumente encontrada no interior do estado associada às formações de Cerrado, floresta estacional e em sua transição com a floresta ombrófila. Devido à inexistência de revisões taxonômicas amplas para os gêneros Adenomera e Pseudopaludicola, optou-se por definir as espécies destes gêneros como "cf." (confer). Isto porque, além destes gêneros caracterizarem-se pela presença de espécies crípticas e outras com alto grau de polimorfismo entre populações (Angulo \& Reichle 2008, Duarte et al. 2010), destacam-se as recentes descrições de espécies para o estado (Berneck et al. 2008, Toledo et al. 2010). 

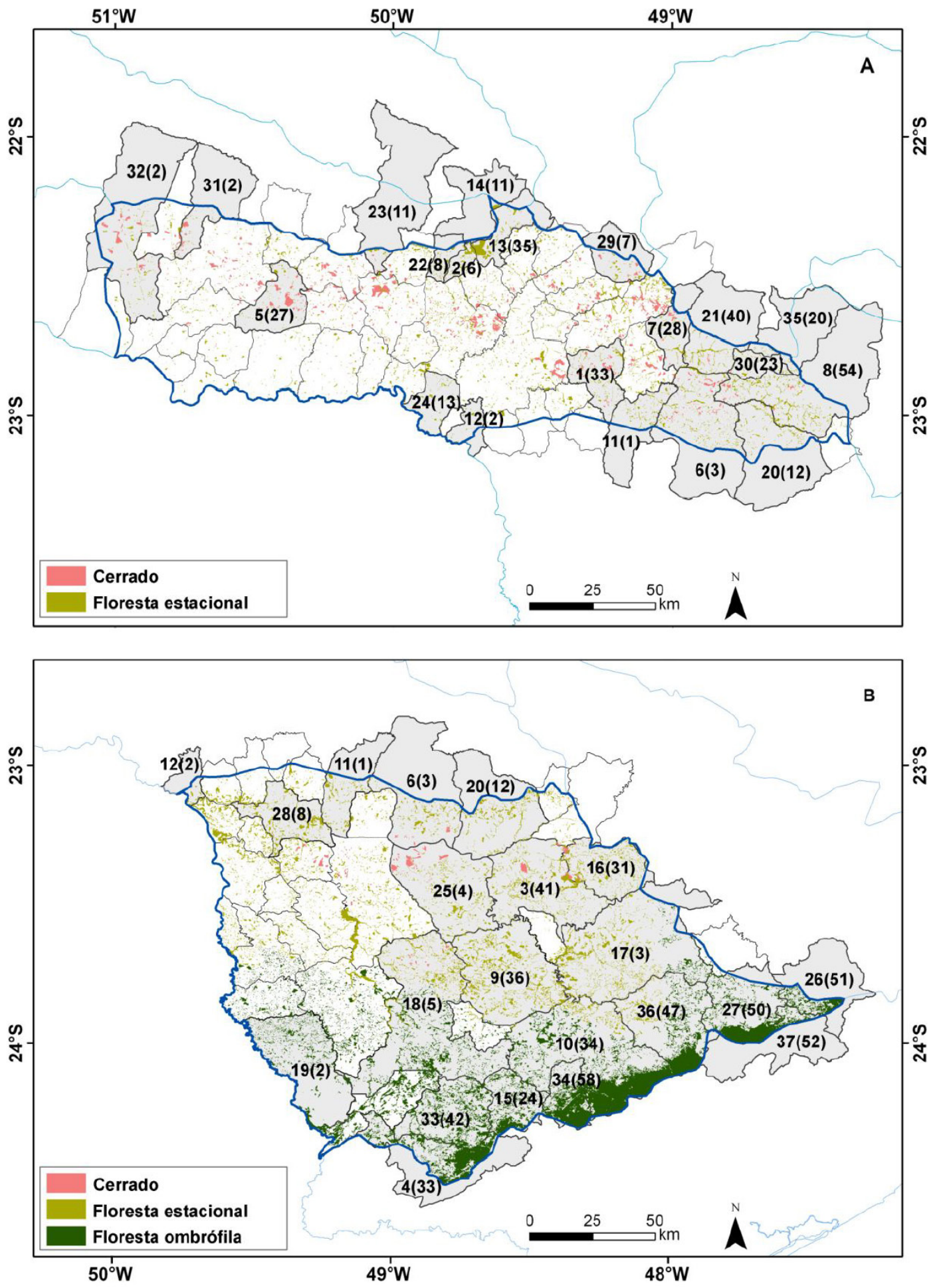

Figura 2. Remanescentes de vegetação natural do Alto (B) e Médio (A) Paranapanema, SP. Os municípios que apresentam registros de anuros em coleções científicas e/ou literatura estão em destaque e o número de espécies registrado para cada um deles encontra-se entre parênteses. Municípios: 1 = Águas de Santa Bárbara, 2 =Alvinlândia, $3=$ Angatuba, $4=$ Apiaí, $5=$ Assis, $6=$ Avaré, $7=$ Borebi, $8=$ Botucatu, $9=$ Buri, $10=$ Capão Bonito, $11=$ Cerqueira César, $12=$ Chavantes, $13=$ Gália, $14=$ Garça, $15=$ Guapiara, $16=$ Guareí, $17=$ Itapetininga, $18=$ Itapeva, $19=$ Itararé, $20=$ Itatinga, $21=$ Lençóis Paulista, $22=$ Lupércio, 23 = Marília, $24=$ Ourinhos, $25=$ Paranapanema, $26=$ Piedade, $27=$ Pilar do Sul, $28=$ Piraju, $29=$ Piratininga, $30=$ Pratânia, $31=$ Quatá 32 = Rancharia, 33 = Ribeirão Branco, 34 = Ribeirão Grande, 35 = São Manuel, 36 = São Miguel Arcanjo, 37 = Tapiraí.

Figure 2. Natural vegetation remnants of Upper (B) and Middle (A) Paranapanema, São Paulo state, Brazil. The municipalities that have records of anurans in scientic collections and/or in the literature are highlighted and the number of species recorded for each one is between parentheses. Municipalities: $1=$ Águas de Santa Bárbara, 2 = Alvinlândia, $3=$ Angatuba, $4=$ Apiaí, $5=$ Assis, $6=$ Avaré, $7=$ Borebi, $8=$ Botucatu, $9=$ Buri, $10=$ Capão Bonito, $11=$ Cerqueira César, $12=$ Chavantes, $13=$ Gália, $14=$ Garça, $15=$ Guapiara, $16=$ Guareí, $17=$ Itapetininga, $18=$ Itapeva, $19=$ Itararé, $20=$ Itatinga, $21=$ Lençóis Paulista, $22=$ Lupércio, 23 = Marília, $24=$ Ourinhos, $25=$ Paranapanema, $26=$ Piedade, $27=$ Pilar do Sul, 28 = Piraju, $29=$ Piratininga, $30=$ Pratânia, $31=$ Quatá, 32 = Rancharia, 33 = Ribeirão Branco, 34 = Ribeirão Grande, 35 = São Manuel, 36 = São Miguel Arcanjo, 37 = Tapiraí. 
A citação das espécies foi feita prioritariamente com base nos dados oriundos de coleções biológicas (CFBH, DZSJRP, MZUSP, ZUEC). Na ausência destes registros, foram consideradas as informações presentes em artigos científicos, livros, dissertações e teses (Apêndice 1). Optou-se pela utilização do sistema de classificação proposta por Pyron \& Wiens (2011) que atualmente engloba o maior número de táxons de anuros e o status de conservação das espécies foi obtido na lista vermelha internacional de espécies ameaçadas de extinção (International... 2013), livro vermelho da fauna brasileira ameaçada de extinção (Machado et al. 2008) e lista de anfíbios ameaçados do estado de São Paulo (Garcia et al. 2009). As informações sobre a ocorrência das espécies nas fitofisionomias presentes no estado (Tabela 1) foram obtidas pela consulta à literatura (Brasileiro et al. 2005, Serafim et al. 2008, Araujo et al. 2009a, 2010, 2013, Condez et al. 2009, Santos et al. 2009, Brassaloti et al. 2010, Forlani et al. 2010, Araujo \& Almeida-Santos 2011, 2013, Maffei et al. 2011a,b, Rossa-Feres et al. 2011).

\section{Resultados e Discussão}

Registrou-se nos municípios do Alto e Médio Paranapanema um total de 124 espécies de anuros pertencentes a doze famílias: Brachycephalidae (5 espécies), Bufonidae (5), Centrolenidae (2), Ceratophryidae (1), Craugastoridae (1), Cycloramphidae (2),

Tabela 1. Espécies de anuros registradas nos municípios do Alto e Médio Paranapanema, SP. Formações vegetais de ocorrência das espécies (CE = fisionomias de Cerrado, FES = floresta estacional semidecidual e FO = floresta ombrófila). Foram considerados prioritariamente os dados oriundos de coleções herpetológicas (CFBH, DZSJRP, MZUSP e ZUEC =1). Na ausência destes registros, utilizaram-se informações contidas em publicações científicas (2) e dissertações e teses (3).

Table 1. Anuran species recorded in the municipalities of Upper and Middle Paranapanema, São Paulo state, Brazil. Vegetation formations (CE = Cerrado physiognomies, $\mathrm{FES}=$ seasonal semideciduous forest and $\mathrm{FO}=$ ombrophilous forest). We considered primarily the data from herpetological collections ( $\mathrm{CFBH}$, DZSJRP, MZUSP and ZUEC = 1). In the absence of these records, we used informations contained in scientific publications (2) and dissertations and theses (3).

\begin{tabular}{|c|c|c|c|}
\hline \multirow[t]{2}{*}{ Família/Espécie } & \multirow{2}{*}{$\begin{array}{c}\text { Fomação } \\
\text { vegetal }\end{array}$} & \multicolumn{2}{|c|}{ Bacia Hidrográfica } \\
\hline & & Alto & Médio \\
\hline \multicolumn{4}{|l|}{ Brachycephalidae } \\
\hline Brachycephalus hermogenesi (Giaretta \& Sawaya 1998) & $\mathrm{FO}$ & 1 & - \\
\hline Ischnocnema cf. hoehnei (B. Lutz 1959 “1958”) & $\mathrm{FO}$ & 1 & - \\
\hline Ischnocnema cf. spanios (Heyer 1985) & $\mathrm{FO}$ & 1 & - \\
\hline Ischnocnema guentheri (Steindachner 1864) & $\mathrm{FO}$ & 1 & - \\
\hline Ischnocnema parva (Girard 1853) & $\mathrm{FO}$ & 1 & - \\
\hline \multicolumn{4}{|l|}{ Bufonidae } \\
\hline Dendrophryniscus brevipollicatus Jiménez de la Espada 1871 “1870” & $\mathrm{FO}$ & 1 & - \\
\hline Rhinella hoogmoedi (Caramaschi \& Pombal 2006) & $\mathrm{FO}$ & 1 & - \\
\hline Rhinella icterica (Spix 1824) & $\mathrm{FES} / \mathrm{FO}$ & 1 & 3 \\
\hline Rhinella ornata (Spix 1824) & $\mathrm{CE} / \mathrm{FES} / \mathrm{FO}$ & 1 & 1 \\
\hline Rhinella schneideri (Werner 1894) & $\mathrm{CE} / \mathrm{FES} / \mathrm{FO}$ & 1 & 1 \\
\hline \multicolumn{4}{|l|}{ Centrolenidae } \\
\hline Vitreorana eurygnatha (A. Lutz 1925) & $\mathrm{FO}$ & 1 & - \\
\hline Vitreorana uranoscopa (Müller 1924) & $\mathrm{FES} / \mathrm{FO}$ & 1 & 1 \\
\hline \multicolumn{4}{|l|}{ Ceratophryidae } \\
\hline Ceratophrys aurita (Raddi 1823) & $\mathrm{FO}$ & 1 & - \\
\hline \multicolumn{4}{|l|}{ Craugastoridae } \\
\hline Haddadus binotatus (Spix 1824) & $\mathrm{CE} / \mathrm{FES} / \mathrm{FO}$ & 1 & 1 \\
\hline \multicolumn{4}{|l|}{ Cycloramphidae } \\
\hline Cycloramphus acangatan Verdade \& Rodrigues $2003^{\mathrm{VU}(\mathrm{IUCN})}$ & $\mathrm{FO}$ & 1 & - \\
\hline Cycloramphus eleutherodactylus (Miranda-Ribeiro 1920) & $\mathrm{FO}$ & 1 & - \\
\hline \multicolumn{4}{|l|}{ Odontophrynidae } \\
\hline Macrogenioglottus alipioi Carvalho 1946 & $\mathrm{FO}$ & 1 & - \\
\hline Odontophrynus americanus (Duméril \& Bibron 1841) & $\mathrm{CE} / \mathrm{FES} / \mathrm{FO}$ & 1 & 1 \\
\hline Odontophrynus cf. cultripes (Reinhardt \& Lütken 1862) & $\mathrm{CE} / \mathrm{FES}$ & - & 1 \\
\hline Proceratophrys boiei (Wied-Neuwied 1824) & $\mathrm{FES} / \mathrm{FO}$ & 1 & 3 \\
\hline Proceratophrys melanopogon (Miranda-Ribeiro 1926) & $\mathrm{FO}$ & 1 & - \\
\hline Proceratophrys moratoi (Jim \& Caramaschi 1980) ${ }^{\mathrm{CA}(\mathrm{IUCN}) ; \mathrm{VU}(\mathrm{SP})}$ & $\mathrm{CE}$ & 2 & 1 \\
\hline \multicolumn{4}{|l|}{ Hemiphractidae } \\
\hline Flectonotus fissilis (Miranda-Ribeiro 1920) & FO & 1 & - \\
\hline Flectonotus ohausi (Wandolleck 1907) & $\mathrm{FO}$ & 1 & - \\
\hline Gastrotheca microdiscus (Andersson in Lönnberg \& Andersson 1910) & $\mathrm{FO}$ & 1 & - \\
\hline \multicolumn{4}{|l|}{ Hylidae } \\
\hline Aplastodiscus albosignatus (A. Lutz \& B. Lutz 1938) & $\mathrm{FO}$ & 1 & - \\
\hline Aplastodiscus callipygius (Cruz \& Peixoto 1985 “1984”) & $\mathrm{FO}$ & 1 & - \\
\hline
\end{tabular}


Tabela 1. Continuação...

Table 1. Continued...

\begin{tabular}{|c|c|c|c|}
\hline \multirow[t]{2}{*}{ Família/Espécie } & \multirow{2}{*}{$\begin{array}{c}\text { Fomação } \\
\text { vegetal }\end{array}$} & \multicolumn{2}{|c|}{ Bacia Hidrográfica } \\
\hline & & Alto & Médio \\
\hline Aplastodiscus cf. ehrhardti (Müller 1924) & FO & 1 & - \\
\hline Aplastodiscus leucopygius (Cruz \& Peixoto 1985 “1984”) & FO & 2 & - \\
\hline Aplastodiscus perviridis A. Lutz in B. Lutz 1950 & $\mathrm{CE} / \mathrm{FES} / \mathrm{FO}$ & 1 & 1 \\
\hline Bokermannohyla astartea (Bokermann 1967) & FO & 1 & - \\
\hline Bokermannohyla circumdata (Cope 1871) & $\mathrm{FES} / \mathrm{FO}$ & 1 & - \\
\hline Bokermannohyla hylax (Heyer 1985) & FO & 1 & - \\
\hline Bokermannohyla izecksohni (Jim \& Caramaschi 1979) ${ }^{\mathrm{CA}(\mathrm{IUCN})}$ & $\mathrm{FES} / \mathrm{FO}$ & - & 1 \\
\hline Bokermannohyla luctuosa (Pombal \& Haddad 1993) & FO & 1 & - \\
\hline Dendropsophus anceps (A. Lutz 1929) & $\mathrm{FES} / \mathrm{FO}$ & 1 & 2 \\
\hline Dendropsophus elegans (Wied-Neuwied 1824) & $\mathrm{FES} / \mathrm{FO}$ & 1 & - \\
\hline Dendropsophus elianeae (Napoli \& Caramaschi 2000) & $\mathrm{CE} / \mathrm{FES}$ & 1 & 1 \\
\hline Dendropsophus giesleri (Mertens 1950) & FO & 1 & - \\
\hline Dendropsophus jimi (Napoli \& Caramaschi 1999) & $\mathrm{CE}$ & 1 & 1 \\
\hline Dendropsophus microps (Peters 1872) & $\mathrm{FES} / \mathrm{FO}$ & 1 & 2 \\
\hline Dendropsophus minutus (Peters 1872) & $\mathrm{CE} / \mathrm{FES} / \mathrm{FO}$ & 1 & 1 \\
\hline Dendropsophus nanus (Boulenger 1889) & $\mathrm{CE} / \mathrm{FES}$ & 1 & 1 \\
\hline Dendropsophus sanborni (Schmidt 1944) & $\mathrm{CE} / \mathrm{FES} / \mathrm{FO}$ & 1 & 1 \\
\hline Dendropsophus seniculus (Cope 1868) & FO & 1 & - \\
\hline Dendropsophus werneri (Cochran 1952) & FO & 2 & - \\
\hline Hypsiboas albomarginatus (Spix 1824) & $\mathrm{FES} / \mathrm{FO}$ & 1 & - \\
\hline Hypsiboas albopunctatus (Spix 1824) & $\mathrm{CE} / \mathrm{FES} / \mathrm{FO}$ & 1 & 1 \\
\hline Hypsiboas bischoffi (Boulenger 1887) & $\mathrm{FES} / \mathrm{FO}$ & 1 & - \\
\hline Hypsiboas caingua (Carrizo 1991 “1990”) & $\mathrm{CE} / \mathrm{FES} / \mathrm{FO}$ & 1 & 1 \\
\hline Hypsiboas caipora Antunes, Faivovich \& Haddad 2008 & FO & 1 & - \\
\hline Hypsiboas faber (Wied-Neuwied 1821) & $\mathrm{CE} / \mathrm{FES} / \mathrm{FO}$ & 1 & 1 \\
\hline Hypsiboas lundii (Burmeister 1856) & $\mathrm{CE} / \mathrm{FES}$ & 1 & 1 \\
\hline Hypsiboas pardalis (Spix 1824) & $\mathrm{FES} / \mathrm{FO}$ & 1 & 1 \\
\hline Hypsiboas prasinus (Burmeister 1856) & $\mathrm{FES} / \mathrm{FO}$ & 1 & 1 \\
\hline Hypsiboas raniceps Cope 1862 & $\mathrm{CE} / \mathrm{FES}$ & - & 1 \\
\hline Hypsiboas semilineatus (Spix 1824) & FO & 2 & - \\
\hline Itapotihyla langsdorffii (Duméril \& Bibron 1841) & $\mathrm{CE} / \mathrm{FES} / \mathrm{FO}$ & 1 & 1 \\
\hline Phasmahyla cochranae (Bokermann 1966) & FO & 1 & - \\
\hline Phyllomedusa distincta A. Lutz in B. Lutz 1950 & FO & 1 & - \\
\hline Phyllomedusa tetraploidea Pombal \& Haddad 1992 & $\mathrm{CE} / \mathrm{FES}$ & 1 & 1 \\
\hline Pseudis platensis Gallardo 1961 & $\mathrm{CE} / \mathrm{FES}$ & 1 & 1 \\
\hline Scinax alter (B. Lutz 1973) & FO & 1 & - \\
\hline Scinax berthae (Barrio 1962) & $\mathrm{CE} / \mathrm{FES} / \mathrm{FO}$ & 1 & 1 \\
\hline Scinax brieni (De Witte 1930) & FO & 1 & - \\
\hline Scinax caldarum (B. Lutz 1968) & $\mathrm{FO}$ & 1 & - \\
\hline Scinax cf. similis (Cochran 1952) & $\mathrm{CE} / \mathrm{FES}$ & - & 1 \\
\hline Scinax crospedospilus (A. Lutz 1925) & $\mathrm{FO}$ & 1 & - \\
\hline Scinax eurydice (Bokermann 1968) & $\mathrm{FO}$ & 1 & - \\
\hline Scinax fuscomarginatus (A. Lutz 1925) & $\mathrm{CE} / \mathrm{FES} / \mathrm{FO}$ & 1 & 1 \\
\hline Scinax fuscovarius (A. Lutz 1925) & $\mathrm{CE} / \mathrm{FES} / \mathrm{FO}$ & 1 & 1 \\
\hline Scinax hayii (Barbour 1909) & FO & 1 & - \\
\hline Scinax hiemalis (Haddad \& Pombal 1987) & $\mathrm{FES} / \mathrm{FO}$ & - & 1 \\
\hline Scinax littoralis (Pombal \& Gordo 1991) & FO & 1 & - \\
\hline Scinax obtriangulatus (B. Lutz 1973) & FO & 2 & - \\
\hline Scinax perereca Pombal, Haddad \& Kasahara 1995 & $\mathrm{FES} / \mathrm{FO}$ & 1 & 2 \\
\hline Scinax perpusillus (A. Lutz \& B. Lutz 1939) & FO & 1 & - \\
\hline Scinax rizibilis (Bokermann 1964) & $\mathrm{FES} / \mathrm{FO}$ & 1 & 1 \\
\hline
\end{tabular}
$\mathrm{FES} / \mathrm{FO}$ vulnerável segundo a lista vermelha internacional de espécies ameaçadas de extinção (International... 2013). vU (SP) Espécie vulnerável segundo a lista de anfíbios ameaçados do estado de São Paulo (Garcia et al. 2009). 
Tabela 1. Continuação...

Table 1. Continued..

\begin{tabular}{|c|c|c|c|}
\hline \multirow[t]{2}{*}{ Família/Espécie } & \multirow{2}{*}{$\begin{array}{c}\text { Fomação } \\
\text { vegetal }\end{array}$} & \multicolumn{2}{|c|}{ Bacia Hidrográfica } \\
\hline & & Alto & Médio \\
\hline Scinax squalirostris (A. Lutz 1925) & $\mathrm{CE} / \mathrm{FES}$ & 1 & 1 \\
\hline Scinax x-signatus (aff. similis) (Spix 1824) & $\mathrm{CE} / \mathrm{FES}$ & - & 1 \\
\hline Sphaenorhynchus caramaschii Toledo, Garcia, Lingnau \& Haddad 2007 & $\mathrm{FES} / \mathrm{FO}$ & 1 & 1 \\
\hline Trachycephalus imitatrix (Miranda-Ribeiro 1926) & FO & 1 & - \\
\hline Trachycephalus lepidus (Pombal, Haddad \& Cruz 2003) & FO & 1 & - \\
\hline Trachycephalus mesophaeus (Hensel 1867) & FO & 1 & - \\
\hline Trachycephalus typhonius (Linnaeus 1758) & $\mathrm{CE} / \mathrm{FES}$ & 1 & 1 \\
\hline \multicolumn{4}{|l|}{ Hylodidae } \\
\hline Crossodactylus caramaschii Bastos \& Pombal 1995 & FES/FO & 1 & 1 \\
\hline Crossodactylus sp. (gr. gaudichaudii) & FES & - & 1 \\
\hline Hylodes cf. asper (Müller 1924) & FO & 1 & - \\
\hline Hylodes cf. cardosoi Lingnau, Canedo \& Pombal 2008 & FO & 1 & - \\
\hline Hylodes heyeri Haddad, Pombal \& Bastos 1996 & FO & 1 & - \\
\hline Hylodes phyllodes Heyer \& Cocroft 1986 & FO & 2 & - \\
\hline Megaelosia cf. goeldii (Baumann 1912) & FO & 2 & - \\
\hline \multicolumn{4}{|l|}{ Leptodactylidae } \\
\hline Adenomera cf. bokermanni Heyer 1973 & FES/FO & 1 & 1 \\
\hline Adenomera cf. marmorata (Steindachner 1867) & $\mathrm{FES} / \mathrm{FO}$ & 1 & 1 \\
\hline Adenomera sp. & FES & 1 & - \\
\hline Eupemphix nattereri Steindachner 1863 & $\mathrm{CE} / \mathrm{FES}$ & 1 & 1 \\
\hline Leptodactylus chaquensis Cei 1950 & $\mathrm{CE} / \mathrm{FES}$ & - & 2 \\
\hline Leptodactylus flavopictus A. Lutz 1926 & FO & 1 & - \\
\hline Leptodactylus furnarius Sazima \& Bokermann 1978 & $\mathrm{CE} / \mathrm{FES}$ & 3 & 1 \\
\hline Leptodactylus fuscus (Schneider 1799) & $\mathrm{CE} / \mathrm{FES} / \mathrm{FO}$ & 1 & 1 \\
\hline Leptodactylus jolyi Sazima \& Bokermann 1978 & $\mathrm{CE}$ & 1 & 1 \\
\hline Leptodactylus labyrinthicus (Spix 1824) & $\mathrm{CE} / \mathrm{FES}$ & 1 & 1 \\
\hline Leptodactylus latrans (Steffen 1815) & $\mathrm{CE} / \mathrm{FES} / \mathrm{FO}$ & 1 & 1 \\
\hline Leptodactylus mystaceus (Spix 1824) & FES & 1 & 1 \\
\hline Leptodactylus mystacinus (Burmeister 1861) & $\mathrm{CE} / \mathrm{FES} / \mathrm{FO}$ & 1 & 1 \\
\hline Leptodactylus notoaktites Heyer 1978 & FO & 1 & - \\
\hline Leptodactylus podicipinus (Cope 1862) & $\mathrm{CE} / \mathrm{FES}$ & 1 & 1 \\
\hline Paratelmatobius cf. gaigeae (Cochran 1938) ${ }^{\mathrm{VU}(\mathrm{SP})}$ & FO & 2 & - \\
\hline Paratelmatobius sp. (aff. cardosoi) & FO & 1 & - \\
\hline Physalaemus centralis Bokermann 1962 & $\mathrm{CE}$ & 1 & 1 \\
\hline Physalaemus cuvieri Fitzinger 1826 & $\mathrm{CE} / \mathrm{FES} / \mathrm{FO}$ & 1 & 1 \\
\hline Physalaemus lateristriga (Steindachner 1864) & FO & 1 & - \\
\hline Physalaemus marmoratus (Reinhardt \& Lütken 1862 “1861”) & $\mathrm{CE}$ & 1 & 1 \\
\hline Physalaemus olfersii (Lichtenstein \& Martens 1856) & FES/FO & 1 & 1 \\
\hline Physalaemus spiniger (Miranda-Ribeiro 1926) & FO & 1 & - \\
\hline Pseudopaludicola cf. falcipes (Hensel 1867) & $\mathrm{CE}$ & - & 1 \\
\hline $\begin{array}{l}\text { Pseudopaludicola cf. murundu Toledo, Siqueira, Duarte, Veiga-Menoncello, Recco- } \\
\text { Pimentel \& Haddad } 2010\end{array}$ & $\mathrm{CE}$ & - & 1 \\
\hline Pseudopaludicola cf. mystacalis (Cope 1887) & $\mathrm{CE}$ & 1 & 1 \\
\hline Pseudopaludicola cf. saltica (Cope 1887) & $\mathrm{CE}$ & - & 1 \\
\hline \multicolumn{4}{|l|}{ Microhylidae } \\
\hline Chiasmocleis albopunctata (Boettger 1885) & $\mathrm{CE} / \mathrm{FES}$ & 1 & 1 \\
\hline Chiasmocleis leucosticta (Boulenger 1888) & FO & 1 & - \\
\hline Elachistocleis bicolor (Guérin-Menéville 1838) & $\mathrm{CE} / \mathrm{FES}$ & 1 & 1 \\
\hline Elachistocleis cesarii (Miranda-Ribeiro 1920) & $\mathrm{CE} / \mathrm{FES}$ & 1 & 1 \\
\hline Myersiella microps (Duméril \& Bibron 1841) & FO & 1 & - \\
\hline
\end{tabular}

${ }_{\text {CA(IUCN) }}$ Espécie criticamente ameaçada segundo a lista vermelha internacional de espécies ameaçadas de extinção (International... 2013). ${ }^{\text {VU (IUCN) }}$ Espécie vulnerável segundo a lista vermelha internacional de espécies ameaçadas de extinção (International... 2013). VU(SP) Espécie vulnerável segundo a lista de anfíbios ameaçados do estado de São Paulo (Garcia et al. 2009). 
Odontophrynidae (6), Hemiphractidae (3), Hylidae (60), Hylodidae (7), Leptodactylidae (27) e Microhylidae (5) (Tabela 1). Foram reunidos mais de 2.700 registros nas coleções biológicas, em um total de 116 espécies determinadas taxonomicamente (Apêndice 1). Adicionalmente, foram incorporadas à listagem algumas espécies presentes apenas na literatura: Aplastodiscus leucopygius, Dendropsophus werneri, Hylodes phyllodes, Hypsiboas semilineatus, Leptodactylus chaquensis, Megaelosia cf. goeldii, Paratelmatobius cf. gaigeae e Scinax obtriangulatus (Bertoluci 2001, Bertoluci \& Rodrigues 2002, Condez et al. 2009, Brassaloti et al. 2010) (Apêndice 1).

Os resultados obtidos neste estudo apontam que ainda existem lacunas de conhecimento sobre esta taxocenose em grande parte dos municípios do Alto e Médio Paranapanema (Figura 2). Dos 91 municípios integral ou parcialmente incluídos nestas bacias hidrográficas, 37 municípios apresentam algum registro de espécie de anuro (Figura 2). Apenas 20 municípios podem ser considerados razoavelmente amostrados, apresentando no mínimo 20 registros de espécies e destes, 15 municípios podem ser classificados como relativamente bem amostrados, tendo sido estudados por longos períodos e/ou vários pesquisadores (Figura 2). No Médio Paranapanema nota-se que a escassez de registros é muito acentuada nas porções central e oeste desta bacia hidrográfica, sendo bem conhecidas apenas as taxocenoses presentes nos municípios de Assis (Ribeiro-Júnior \& Bertoluci 2009, Araujo \& Almeida-Santos 2011) e Gália (Brassaloti et al. 2010) (Figura 2). Os municípios melhor amostrados estão concentrados na porção mais a leste, como Águas de Santa Bárbara (Araujo et al. 2013), Borebi (Maffei et al. 2011b), Lençóis Paulista (Maffei et al. 2011a) e Botucatu (Jim 2002). Destes, apenas Botucatu pode ser considerado exaustivamente inventariado por diversos pesquisadores ao longo de décadas (Jim 1980, 2002, Rossa-Feres \& Jim 1994, Rolim 2009) (Figura 2). Em relação ao Alto Paranapanema, percebe-se que os municípios presentes nas regiões sul e sudeste, que apresentam predomínio de floresta ombrófila, são os mais adequadamente amostrados, como Piedade e Tapiraí (Condez et al. 2009), Pilar do Sul (Antunes 2007), Ribeirão Branco, Ribeirão Grande (Bertoluci 2001) e São Miguel Arcanjo (Forlani et al. 2010) (Figura 2). Entre os municípios com predominância de floresta estacional semidecidual e remanescentes de Cerrado, apenas Angatuba, Guareí (Lopes 2010, Araujo \& Almeida-Santos 2013) e Buri (V. K. Verdade, comunicação pessoal) podem ser considerados apropriadamente inventariados. Assim como observado no Médio Paranapanema, a região oeste do Alto Paranapanema pode ser considerada como uma grande lacuna de conhecimento deste táxon (Figura 2).

A riqueza de espécies de anuros atribuída ao Alto e Médio Paranapanema provavelmente está subestimada. Além da existência de exemplares não identificados nas coleções científicas que podem representar espécies ainda não descritas, deve-se considerar que estudos filogenéticos que incluam análises moleculares podem identificar novas espécies nestas bacias hidrográficas, sobretudo em gêneros que apresentam espécies crípticas como Adenomera (Angulo \& Reichle 2008, Berneck et al. 2008) e Pseudopaludicola (Duarte et al. 2010, Toledo et al. 2010). O número de espécies registrado no Alto Paranapanema (113 espécies) foi muito superior ao encontrado no Médio Paranapanema (64 espécies). Dentre os anuros registrados no Alto Paranapanema, 48\% das espécies foram encontradas exclusivamente nos municípios com predomínio de floresta ombrófila. Conhecidamente, a fauna de anuros presente nesta fitofisionomia apresenta elevado endemismo e altas taxas de especiação em suas comunidades (Cruz \& Feio 2007, Carnaval et al. 2009). Assim como ocorre no estado (Rossa-Feres et al. 2011), é possível identificar uma diminuição gradual na riqueza de espécies partindo das porções sul e sudeste do Alto Paranapanema, onde predominam a floresta ombrófila, em direção ao Médio Paranapanema que apresenta formações de Cerrado e floresta estacional semidecidual.

A maior parte das espécies reunidas neste estudo (54 espécies; 43,5\%) ocorre exclusivamente em floresta ombrófila e estão presentes apenas no Alto Paranapanema. Apenas três espécies $(2,5 \%)$ são restritas às localidades de floresta estacional semidecidual destas bacias hidrográficas: Adenomera sp., Crossodactylus sp. (gr. gaudichaudii) e Leptodactylus mystaceus. Vinte espécies (16\%) estão associadas a essas duas formações florestais. Foi possível observar que Adenomera cf. marmorata, Bokermannohyla circumdata, Dendropsophus elegans, Hypsiboas albomarginatus, H. bischoffi, H. pardalis, Proceratophrys boiei e Rhinella icterica, espécies consideradas típicas de floresta ombrófila (Haddad et al. 2008b, Araujo et al. 2010, Forlani et al. 2010, Rossa-Feres et al. 2011), apresentam uma ocorrência ocasional na floresta estacional semidecidual, estando principalmente distribuídas na transição entre estas duas fitofisionomias que ocorre predominantemente na porção sul e sudeste do Alto Paranapanema. Outras como Crossodactylus caramaschii, Dendropsophus microps, Scinax hiemalis, S. perereca e $S$. rizibilis estão associadas a ambas fitofisionomias, sendo que o baixo número de registros destas espécies na floresta estacional semidecidual do estado pode estar relacionada com os processos históricos de uso e ocupação do solo no Planalto Ocidental Paulista. Dentre as formações florestais brasileiras esta é, sem dúvida, a que sofreu maior desmatamento (Leitão-Filho 1987, Durigan et al. 2000, Ribeiro et al. 2009). A intensa fragmentação desta fitofisionomia levou a perda e desconexão de hábitats, propiciando a degradação de corpos d'água que são utilizados como sítios reprodutivos por muitas espécies de anuros, interferindo diretamente no ciclo reprodutivo destas espécies e propiciando o declínio e desaparecimento de populações (Becker et al. 2007).

Somente nove espécies $(7,3 \%)$ foram encontradas exclusivamente em formações de Cerrado. Estas espécies ocorrem predominantemente nas formações campestres e savânicas, que são as fisionomias vegetais mais vulneráveis ao desaparecimento no estado de São Paulo (Durigan et al. 2003, Durigan \& Ratter 2006). O baixo número de registros de espécies como Leptodactylus jolyi, Pseudopaludicola cf. falcipes, $P$. cf. murundu, $P$. cf. mystacalis, $P$. cf. saltica e Proceratophrys moratoi pode ser reflexo da pequena porção que esta formação vegetal ocupa atualmente nos municípios do Alto e Médio Paranapanema. Possivelmente, estas populações representam o limite de distribuição mais austral das espécies neste domínio fitogeográfico (Diniz-Filho et al. 2008, Valdujo 2011) e a conservação e conexão dos remanescentes de Cerrado nestas bacias hidrográficas podem contribuir para a manutenção da diversidade genética destes anuros. Alguns estudos apontam uma grande similaridade faunística entre as taxocenoses de anuros que ocorrem em áreas de Cerrado e floresta estacional semidecidual (Araujo et al. 2009a, Santos et al. 2009, Vasconcelos et al. 2011). Neste inventário foram registradas 19 espécies $(15,3 \%)$ em ambas as formações vegetais. Esta similaridade pode ser atribuída ao fato da floresta estacional semidecidual no estado possuir características de descontinuidade, apresentando-se permeada por manchas dispersas de Cerrado em suas diversas fitofisionomias (Leitão-Filho 1987, Durigan et al. 2004b).

Algumas espécies amplamente encontradas no Alto e Médio Paranapanema (16 espécies; 13\%) podem ser consideradas mais generalistas no uso do ambiente, ocorrendo em todas as formações vegetais analisadas e sendo comumente encontradas em várias regiões do Brasil como Dendropsophus minutus, D. nanus, Hypsiboas albopunctatus, Leptodactylus fuscus, L. mystacinus, Physalaemus cuvieri, Rhinella schneideri, Scinax fuscomarginatus e S. fuscovarius (Haddad et al. 2008b, Frost 2013). 
Dentre as espécies registradas, Bokermannohyla izecksohni e Proceratophrys moratoi são consideradas criticamente ameaçadas e Cycloramphus acangatan é classificada como vulnerável pela International Union for Conservation of Nature - IUCN (International... 2013). Proceratophrys moratoi e Paratelmatobius cf. gaigeae são apontadas como vulneráveis no estado de São Paulo (Garcia et al. 2009). Estas espécies apresentam distribuição restrita ao estado de São Paulo (Haddad et al. 2008b, Frost 2013), exceto Paratelmatobius cf. gaigeae que está associada à Serra da Bocaina e Serra do Mar (Garcia et al. 2009, Frost 2013). O único registro desta espécie no Alto Paranapanema é oriundo do município de Ribeirão Grande (Bertoluci 2001) e deve ser visto com cautela, já que não foi confirmado pela existência de exemplares depositados nas coleções analisadas. Além de ocorrer nos municípios do Alto e Médio Paranapanema, como Avaré, Botucatu e Lençóis Paulista (Maffei 2010, Maffei et al. 2011a,c), Proceratophrys moratoi é encontrada em Bauru, Brotas e São Carlos (Rolim 2009, Maffei et al. 2011c). Bokermannohyla izecksohni, que no Médio Paranapanema ocorre apenas no município de Botucatu, também está presente nos municípios de Cotia e Itanhaém (Frost 2013). A espécie Cycloramphus acangatan é encontrada na Serra de Paranapiacaba (Condez et al. 2009, Frost 2013), incluindo alguns municípios do Alto Paranapanema como Guapiara, Piedade, Pilar do Sul, Ribeirão Grande, São Miguel Arcanjo e Tapiraí.

Este estudo evidencia a carência de registros de anuros para grande parte dos municípios do Alto e Médio Paranapanema, o que reforça a necessidade da realização de mais inventários para ampliar o conhecimento sobre a composição e distribuição das espécies nesta região do estado. As informações reunidas em estudos como este podem contribuir na avaliação do status de conservação das espécies e na elaboração de medidas efetivas de conservação destas populações (Verdade et al. 2012).

\section{Agradecimentos}

Somos gratos aos curadores das coleções herpetológicas Célio F. B. Haddad, Denise C. Rossa Feres, Felipe Toledo e Hussam Zaher por permitirem o acesso aos espécimes e registros de campo. Agradecemos à Cinthia A. Brasileiro, Fernando R. Silva, Otavio A. V. Marques, Vanessa K. Verdade e aos dois revisores anônimos pelas sugestões que aprimoraram este manuscrito. Somos gratos ao Instituto Florestal pelo suporte logístico e financeiro. Selma M. Almeida-Santos agradece ao CNPq e a FAPESP pelo apoio financeiro.

\section{Referências Bibliográficas}

AB'SÁBER, A.N. 2005. Os domínios de natureza no Brasil: potencialidades paisagísticas. Ateliê Editorial, São Paulo.

ALMEIDA, S.C., MAFFEI, F., ROLIM, D.C., UBAID, F.K. \& JIM, J. 2008. Amphibia, Anura, Hylidae, Sphaenorhynchus caramaschii: distribution extension in state of São Paulo, Brazil. Check List 4(4):439-441.

ANGULO, A. \& REICHLE, S. 2008. Acoustic signals, species diagnosis, and species concepts: the case of a new cryptic species of Leptodactylus (Amphibia, Anura, Leptodactylidae) from the Chapare region, Bolivia. Zool. J. Linn. Soc. 152:59-77. http://dx.doi.org/10.1111/j.10963642.2007.00338.x

ANTUNES, A.P. 2007. Descrição taxonômica e história natural de uma nova espécie de Hypsiboas da Mata Atlântica do alto da Serra de Paranapiacaba, estado de São Paulo (Amphibia, Anura, Hylidae). Dissertação de Mestrado, Universidade Estadual Paulista, Rio Claro.

ARAUJO, C.O. \& ALMEIDA-SANTOS, S.M. 2011. Herpetofauna de um remanescente de Cerrado no estado de São Paulo, sudeste do Brasil. Biota Neotrop. 11(3): http://www.biotaneotropica.org.br/v11n3/pt/ abstract?article+bn00511032011 (último acesso em 06/06/2012).
ARAUJO, C.O. \& ALMEIDA-SANTOS, S.M. 2013. Composição, riqueza e abundância de anuros em um remanescente de Cerrado e Mata Atlântica no estado de São Paulo. Biota Neotrop. 13(1): http://www.biotaneotropica. org.br/v13n1/pt/abstract?inventory+bn02613012013 (último acesso em 12/07/2013).

ARAUJO, C.O., CONDEZ, T.H. \& SAWAYA, R.J. 2009a. Anfíbios anuros do Parque Estadual das Furnas do Bom Jesus, sudeste do Brasil, e suas relações com outras taxocenoses no Brasil. Biota Neotrop. 9(2): http:// www.biotaneotropica.org.br/v9n2/pt/abstract?article+bn01109022009 (último acesso em 13/02/2010).

ARAUJO, C.O., CONDEZ, T.H., BOVO, R.P., CENTENO, F.C. \& LUIZ, A.M. 2010. Amphibians and reptiles of the Parque Estadual Turístico do Alto Ribeira (PETAR), SP: an Atlantic Forest remnant of southeastern Brazil. Biota Neotrop. 10(4): http://www.biotaneotropica.org.br/v10n4/ pt/abstract?inventory+bn01710042010 (último acesso em 02/03/2011).

ARAUJO, C.O., CORRÊA, D.T. \& ALMEIDA-SANTOS, S.M. 2013. Anuros da Estação Ecológica de Santa Bárbara, um remanescente de formações abertas de Cerrado no estado de São Paulo. Biota Neotrop. 13(3): http://www.biotaneotropica.org.br/v13n3/pt/abstract?inventory+ bn01613032013 (último acesso em 15/08/2013).

ARAÚJO, O.G.S., TOLEDO, L.F., GARCIA, P.C.A. \& HADDAD, C.F.B. 2009b. The amphibians of São Paulo State. Biota Neotrop. 9(4): http://www.biotaneotropica.org.br/v9n4/en/abstract?inventory+ bn03109042009 (último acesso em 20/04/2010).

BECKER, C.G., FONSECA, C.R., HADDAD, C.F.B., BATISTA, R.F. \& PRADO, P.I. 2007. Habitat split and the global decline of amphibians. Science 5857(318):1775-1777. http://dx.doi.org/10.1126/science.1149374

BERNECK, B.V.M., COSTA, C.O.R. \& GARCIA, P.C.A. 2008. A new species of Leptodactylus (Anura: Leptodactylidae) from the Atlantic Forest of São Paulo State, Brazil. Zootaxa 1795:46-56.

BERTOLUCI, J. \& RODRIGUES, M.T. 2002. Utilização de hábitats reprodutivos e micro-hábitats de vocalização em uma taxocenose de anuros (Amphibia) da Mata Atlântica do sudeste do Brasil. Pap. Avulsos de Zool. 42(11):287-297.

BERTOLUCI, J. 2001. Anfíbios anuros. In Intervales (C. Leonel, org.). Fundação Florestal/SEMA, São Paulo, p.159-168.

BRANDÃO, R.A. \& ARAÚJO, A.F.B. 2002. A herpetofauna associada a matas de galeria no Distrito Federal. In Cerrado: caracterização e recuperação de matas de galeria (J.F. Ribeiro, C.E.L. Fonseca \& J.C. Sousa-Silva, orgs.). EMBRAPA/CPAC, Planaltina, p.560-604.

BRASILEIRO, C.A., SAWAYA, R.J., KIEFER, M.C. \& MARTINS, M. 2005. Amphibians of an open Cerrado fragment in southeastern Brazil. Biota Neotrop. 5(2): http://www.biotaneotropica.org.br/v5n2/ pt/abstract?article+BN00405022005 (último acesso em 16/07/2010).

BRASSALOTI, R.A., ROSSA-FERES, D.C. \& BERTOLUCI, J. 2010. Anurofauna da Floresta Estacional Semidecidual da Estação Ecológica dos Caetetus, sudeste do Brasil. Biota Neotrop. 10(1): http://www. biotaneotropica.org.br/v10n1/pt/abstract?inventory+bn01810012010 (último acesso em 16/07/2010).

CARNAVAL, A.C., HICKERSON, M.J., HADDAD, C.F.B., RODRIGUES, M.T. \& MORITZ, C. 2009. Stability predicts genetic diversity in the Brazilian Atlantic Forest Hotspot. Science 323:785-789. http://dx.doi. org/10.1126/science. 1166955

COLLI, G.R., BASTOS, R.P. \& ARAÚJO, A.F.B. 2002. The character and dynamics of the Cerrado herpetofauna. In The cerrados of Brazil: ecology and natural history of a Neotropical Savanna (P.S. Oliveira \& R.J. Marquis, eds.). Columbia University Press, New York, p.223-241.

CONDEZ, T.H., SAWAYA, R.J. \& DIXO, M. 2009. Herpetofauna dos remanescentes de Mata Atlântica da região de Tapiraí e Piedade, SP, sudeste do Brasil. Biota Neotrop. 9(1): http://www.biotaneotropica. org.br/v9n1/en/abstract?inventory+bn01809012009 (último acesso em 20/07/2010).

CRUZ, C.A.G. \& FEIO, R.N. 2007. Endemismos em anfíbios em áreas de altitude na Mata Atlântica no sudeste do Brasil. In Herpetologia no Brasil II (L.B. Nascimento \& M.E. Oliveira, eds.). Sociedade Brasileira de Herpetologia, Belo Horizonte, p.117-126. 
DINIZ-FILHO, J.A.F., BINI, L.M., VIEIRA, C.M., BLAMIRES, D., TERRIBILE, L., BASTOS, R., OLIVEIRA, G. \& BARRETO, B. 2008. Spatial patterns of terrestrial vertebrate species richness in the Brazilian Cerrado. Zool. Stud. 47(2):146-157.

DUARTE, T.C., VEIGA-MENONCELLO, A.C.P., LIMA, J.F.R., STRUSSMANN, C., DEL GRANDE, M.L., GIARETTA, A.A., PEREIRA, E.G., ROSSA-FERES, D.C. \& RECCO-PIMENTEL, S.M. 2010. Chromosome analysis in Pseudopaludicola (Anura, Leiuperidae), with description of sex chromosomes XX/XY in P. saltica. Hereditas 147(2):43-52. http://dx.doi.org/10.1111/j.1601-5223.2009.02153.x

DURIGAN, G. \& RATTER, J.A. 2006. Successional changes in Cerrado and Cerrado/forest ecotonal vegetation in Western São Paulo State, Brazil, 1962-2000. Edinburgh J. Bot. 63(1):119-130. http://dx.doi.org/10.1017/ S0960428606000357

DURIGAN, G. 2010. Plano de manejo da Estação Ecológica de Assis. Instituto Florestal/SEMA, São Paulo.

DURIGAN, G., FRANCO, G.A.D.C. \& SIQUEIRA, M.F. 2004a. A vegetação dos remanescentes de cerrado no Estado de São Paulo. In Viabilidade da conservação dos remanescentes de Cerrado no Estado de São Paulo (M.D. Bittencourt \& R.R. Mendonça, orgs.). Annablume, FAPESP, São Paulo, p.29-56.

DURIGAN, G., FRANCO, G.A.D.C., SAITO, M. \& BAITELO, J.B. 2000. Estrutura e diversidade do componente arbóreo da floresta na Estação Ecológica dos Caetetus, Gália, SP. Rev. Bras. Bot. 23(4):371-383. http:// dx.doi.org/10.1590/S0100-84042000000400003

DURIGAN, G., SIQUEIRA, M.F. \& FRANCO, G.A.D.C. 2007. Threats to the Cerrado remnants of the State of São Paulo, Brazil. Braz. Sci. Agric. 64(4):355-363.

DURIGAN, G., SIQUEIRA, M.F., FRANCO, G.A.D.C. \& CONTIERI, W.A. 2004b. A flora arbustivo-arbórea do Médio Paranapanema: base para a restauração dos ecossistemas naturais. In Pesquisas em conservação e recuperação ambiental no Oeste Paulista: resultados da cooperação Brasil/Japão (O. Vilas-Boas \& G. Durigan, eds.). Páginas e Letras, São Paulo, p.199-239.

DURIGAN, G., SIQUEIRA, M.F., FRANCO, G.A.D.C., BRIDGEWATER, S. \& RATTER, J.A. 2003. The vegetation of priority areas for Cerrado conservation in São Paulo State, Brazil. Edinburgh J. Bot. 60(2):217-241. http://dx.doi.org/10.1017/S0960428603000155

FORLANI, M.C., BERNARDO, P.H., HADDAD, C.F.B. \& ZAHER, H. 2010. Herpetofauna of the Carlos Botelho State Park, São Paulo state, Brazil. Biota Neotrop. 10(3): http://www.biotaneotropica.org.br/v10n3/ en/abstract?inventory+bn00210032010 (último acesso em 06/06/2011).

FROST, D.R. 2013. Amphibian species of the world: an online reference. version 5.6. American Museum of Natural History, New York. http:// research.amnh.org/herpetology/amphibia/index.html (último acesso em 06/02/2013).

GARCIA, P.C.A., SAWAYA, R.J., MARTINS, I.A., BRASILEIRO, C.A., VERDADE, V.K., JIM, J., SEGALLA, M.V., MARTINS, M., ROSSAFERES, D.C., HADDAD, C.F.B., TOLEDO, L.F., PRADO, C.P.A., BERNECK, B.M. \& ARAÚJO, O.G.S. 2009. Anfíbios. In Fauna ameaçada de extinção no estado de São Paulo: vertebrados (M. Bressan, M.C.M. Kierulff \& A.M. Sugieda, orgs.). Fundação Parque Zoológico de São Paulo/SEMA, São Paulo, p.329-347.

HADDAD, C.F.B. \& PRADO, C.P.A. 2005. Reproductive modes in frogs and their unexpected diversity in the Atlantic Forest of Brazil. BioScience 55(3):207-217. http://dx.doi.org/10.1641/0006-3568(2005)055[0207:RM IFAT]2.0.CO;2

HADDAD, C.F.B., GIOVANELLI, J.G.R. \& ALEXANDRINO, J. 2008a. O aquecimento global e seus efeitos na distribuição e declínios dos anfíbios. In Biologia e mudanças climáticas no Brasil (M.S. Buckeridge, org.). RiMa, São Carlos, p.195-206.

HADDAD, C.F.B., TOLEDO, L.F. \& PRADO, C.P.A. 2008b. Anfíbios da Mata Atlântica: guia dos anfíbios anuros da Mata Atlântica. Editora Neotropica, São Paulo.

INSTITUTO DE PESQUISAS TECNOLÓGICAS - IPT. 1981. Mapa geológico do estado de São Paulo. São Paulo: Série Monografias 6(1):1$126 \mathrm{p}$.

INTERNATIONAL UNION FOR CONSERVATION OF NATURE - IUCN. 2013. The IUCN red list of threatened species. Version 2013.1. http:// www.iucnredlist.org (último acesso em: 12/07/2013).
IOP, S., CALDART, V.M., SANTOS, T.G. \& CECHIN, S.Z. 2011. Anurans of Turvo State Park: testing the validity of Seasonal Forest as a new biome in Brazil. J. Nat. Hist. 45(39-40):2443-2461. http://dx.doi.org/10.1080/ 00222933.2011.596951

JIM, J. 1980. Aspectos ecológicos dos anfíbios registrados na região de Botucatu, São Paulo (Amphibia, Anura). Tese de doutorado, Universidade de São Paulo, São Paulo.

JIM, J. 2002. Distribuição altitudinal e estudo de longa duração de anfíbios da região de Botucatu, estado de São Paulo. Tese de livre-docência, Universidade Estadual Paulista, Botucatu.

KLINK, C.A. \& MACHADO, R.B. 2005. Conservation of the Brazilian Cerrado. Conserv. Biol. 19(3):707-713. http://dx.doi.org/10.1111/j.15231739.2005.00702.x

KRONKA, F.J.N., NALON, M.A. \& MATSUKUMA, C.K. 2005. Inventário florestal da vegetação natural do estado de São Paulo. São Paulo. Instituto Florestal, SEMA, São Paulo.

LEITÃO-FILHO, H.F. 1987. Considerações sobre a florística de florestas tropicais e sub-tropicais do Brasil. I. P. E. F. 35:41-46.

LEPSCH, I.F., SARAIVA, I.R., DONZELI, P.L., MARINHO, M.A., SAKAI, E., GUILLAUMON, J.R., PFEIFER, R.M., MATTOS, I.F.A., ANDRADE, W.J. \& SILVA, C.E.F. 1990. Macrozoneamento das terras da região do rio Ribeira de Iguape, SP. Boletim do IAC 19:1-181.

LOPES, P.C. 2010. Distribuição e abundância de anfíbios e répteis neotropicais em paisagem silvicultural em São Paulo, Brasil. Dissertação de Mestrado, Universidade de São Paulo, Piracicaba.

MACHADO, A.B.M., DRUMMOND, G.M. \& PAGLIA, A.P. 2008. Livro vermelho da fauna brasileira ameaçada de extinção. Fundação Biodiversitas/MMA, Belo Horizonte.

MAFFEI, F. 2010. Diversidade e uso do habitat de comunidades de anfíbios anuros em Lençóis Paulista, estado de São Paulo. Dissertação de Mestrado, Universidade Estadual Paulista, Botucatu.

MAFFEI, F., UBAID, F.K. \& JIM, J. 2011a. Anfíbios: Fazenda Rio Claro, Lençóis Paulista, SP, Brasil. Canal 6, Bauru.

MAFFEI, F., UBAID, F.K. \& JIM, J. 2011b. Anurofauna em área de cerrado aberto no município de Borebi, estado de São Paulo, Sudeste do Brasil: uso do habitat, abundância e variação sazonal. Biota Neotrop. 11(2): http:// www.biotaneotropica.org.br/v11n2/pt/abstract?article+bn04011022011 (último acesso em 06/02/2012).

MAFFEI, F., UBAID, F.K. \& JIM, J. 2011c Discovery of the fifth population of a threatened and endemic toad of the Brazilian Cerrado, Proceratophrys moratoi (Anura, Cycloramphidae). Herpetology Notes 4:95-96.

MAFFEI, F., UBAID, F.K., ALMEIDA, S.C., ROLIM, D.C., SCARPELLINI JUNIOR, D.G., MOYA, G.M., SPIRANDELLI-CRUZ, E.F. \& JIM, J. 2009. Amphibia, Anura, Hylidae, Dendropsophus microps (Peters, 1872): distribution extension in state of São Paulo, Brazil and first record in Cerrado domain. Check List 5(4):776-779.

MITTERMEIER, R.A., GIL, P.R., HOFFMAN, M., PILGRIM, J., BROOKS, T., MITTERMEIER, C.G., LAMOREUX, J. \& FONSECA, G.A.B. 2004. Hotspots revisited: earth's biologically richest and most endangered terrestrial ecoregions. CEMEX \& Agrupacion Sierra Madre, Cidade do México.

MORAES, R.A., SAWAYA, R.J. \& BARRELLA, W. 2007. Composição e diversidade de anfíbios anuros em dois ambientes de Mata Atlântica no Parque Estadual Carlos Botelho, São Paulo, sudeste do Brasil. Biota Neotrop. 7(2):http://www.biotaneotropica.org.br/v7n2/pt/ abstract?article+bn00307022007 (último acesso em 15/07/2009).

MORELLATO, L.P.C. \& HADDAD, C.F.B. 2000. Introduction: the brazilian Atlantic Forest. Biotropica 32(4b):786-792. http://dx.doi. org/10.1111/j.1744-7429.2000.tb00618.x

OLIVEIRA, R.J. 2006. Variação da composição florística e da diversidade alfa das florestas atlânticas no estado de São Paulo. Tese de Doutorado, Universidade Estadual de Campinas, Campinas.

OLIVEIRA-FILHO, A.T. \& FONTES, M.A.L. 2000. Patterns of floristic differentiation among atlantic forests in Southeastern Brazil and the influence of climate. Biotropica 32(4b):793-810. http://dx.doi. org/10.1111/j.1744-7429.2000.tb00619.x

OLIVEIRA-FILHO, A.T. \& RATTER, J.A. 2002. Vegetation physiognomies and woody flora of the Cerrado biome. In The cerrados of Brazil: 
ecology and natural history of a Neotropical Savanna (P.S. Oliveira \& R.J. Marquis, orgs.). Columbia University Press, NewYork, p.91-120.

PEEL, M.C., FINLAYSON, B.L. \& McMAHON, T.A. 2007. Updated world map of the Köppen-Geiger climate classification. Hydrol. Earth Syst. Sci. 11:1633-1644. http://dx.doi.org/10.5194/hess-11-1633-2007

PRADO, D.E. 2000. Seasonally dry forests of Tropical South America: from forgotten ecosystems to a new phytogeographic unit. Edinburgh J. Bot. 57(3):437-461. http://dx.doi.org/10.1017/S096042860000041X

PYRON, R.A. \& WIENS, J.J. 2011. A large-scale phylogeny of Amphibia including over 2800 species, and a revised classification of extant frogs, salamanders, and caecilians. Mol. Phylogenet. Evol. 61(2):543-583. http:// dx.doi.org/10.1016/j.ympev.2011.06.012

RATTER, J.A., RIBEIRO, J.F. \& BRIDGEWATER, S. 1997. The Brazilian Cerrado vegetation and threats to its biodiversity. Ann. Bot. 80:223-230. http://dx.doi.org/10.1006/anbo.1997.0469

RIBEIRO, J.F. \& WALTER, B.M.T. 2008. As principais fitofisionomias do Bioma Cerrado. In Cerrado: ecologia e flora (S.M. Sano, S.P. Almeida \& J.F. Ribeiro, eds.). EMBRAPA, Planaltina, p.153-212.

RIBEIRO, M.C., METZGER, J.P., MARTENSEN, A.C., PONZONI, F.J. \& HIROTA, M.M. 2009. The Brazilian Atlantic Forest: how much is left, and how is the remaining forest distributed? Implications for conservation. Biol. Conserv. 142:1144-1156. http://dx.doi.org/10.1016/j. biocon.2009.02.021

RIBEIRO-JÚNIOR, J.W. \& BERTOLUCI, J. 2009. Anuros do cerrado da Estação Ecológica e da Floresta Estadual de Assis, sudeste do Brasil. Biota Neotrop. 9(1): http://www.biotaneotropica.org.br/v9n1/pt/abstrac t?inventory+bn02709012009 (último acesso em 16/07/2010).

ROLIM, D.C. 2009. Bioecologia de Odontophrynus moratoi (Amphibia, Anura, Cycloramphidae). Dissertação de Mestrado, Universidade Estadual Paulista, Botucatu.

ROSS, J.L.S. \& MOROZ, I.C. 1997. Mapa geomorfológico do estado de São Paulo. FFLCH-USP/IPT/FAPESP, São Paulo.

ROSSA-FERES, D.C. \& JIM, J. 1994. Distribuição sazonal em comunidades de anfíbios anuros na região de Botucatu, São Paulo. Rev. Bras. Biol. 54(2):323-334

ROSSA-FERES, D.C., MARTINS, M., MARQUES, O.A.V., MARTINS, I.A., SAWAYA, R.J. \& HADDAD, C.F.B. 2008. Herpetofauna. In Diretrizes para a conservação e restauração da biodiversidade no estado de São Paulo (R.R. Rodrigues \& V.LR. Bononi, orgs.). Instituto de Botânica, SEMA, São Paulo, p.82-94.

ROSSA-FERES, D.C., SAWAYA, R.J., FAIVOVICH, J., GIOVANELLI, J.G.R., BRASILEIRO, C.A., SCHIESARI, L., ALEXANDRINO, J. \& HADDAD, C.F.B. 2011. Anfíbios do Estado de São Paulo, Brasil: Conhecimento Atual e Perspectivas. Biota Neotrop. 11(1a): http://www. biotaneotropica.org.br/v11n1a/pt/abstract?inventory+bn0041101a2011 (último acesso em 22/02/2012).

SANO, E.E., ROSA, R., BRITO, J.L.S. \& FERREIRA, L.G. 2010. Land cover mapping of the tropical savanna region in Brazil. Environ. Monit. Assess. 166:113-124. http://dx.doi.org/10.1007/s10661-009-0988-4

SANTOS, T.G., VASCONCELOS, T.S., ROSSA-FERES, D.C. \& HADDAD, C.F.B. 2009. Anurans of a seasonally dry tropical forest: Morro do Diabo State Park, São Paulo state, Brazil. J. Nat. Hist. 43(15-16):973-993. http:// dx.doi.org/10.1080/00222930802702498

SEGALLA, M.V., CARAMASCHI, U., CRUZ, C.A.G., GARCIA, P.C.A., GRANT, T., HADDAD, C.F.B \& LANGONE, J. 2012. Brazilian amphibians - List of species. http://www.sbherpetologia.org.br (último acesso em 12/07/2013).

SERAFIM, H., IENNE, S., CICCHI, P.J.P \& JIM, J. 2008. Anurofauna de remanescentes de floresta Atlântica do município de São José do
Barreiro, estado de São Paulo, Brasil. Biota Neotrop. 8(2): http://www. biotaneotropica.org.br/v8n2/pt/abstract?article+bn01008022008 (último acesso em 16/04/2011).

SETZER, J. 1966. Atlas climático e ecológico do estado de São Paulo. Comissão Interestadual da Bacia do Paraná, Uruguai e Centrais Elétricas de São Paulo, São Paulo.

SILVA, F.R., ALMEIDA-NETO, M., PRADO, V.H.M., HADDAD, C.F.B. \& ROSSA-FERES, D.C. 2012. Humidity levels drive reproductive modes and phylogenetic diversity of amphibians in the Brazilian Atlantic Forest. J. Biogeogr. 39(9):1720-1732. http://dx.doi.org/10.1111/j.13652699.2012.02726.x

SILVA, J.M.C. \& BATES, J.M. 2002. Biogeographic patterns and conservation in the South American Cerrado: a tropical savanna hotspot. BioScience 52(3):225-233. http://dx.doi.org/10.1641/0006-3568(2002)052[0225:BP ACIT]2.0.CO;2

SILVANO, D. 2011. Distribuição e conservação de anfíbios no Cerrado em cenários atuais e futuros. Tese de Doutorado, Universidade de Brasília, Brasília.

TOLEDO, L.F., SIQUEIRA, S., DUARTE, T.C., VEIGA-MENONCELLO, A.C.P., RECCO-PIMENTEL, S.M. \& HADDAD, C.F.B. 2010. Description of a new species of Pseudopaludicola Miranda-Ribeiro, 1926 from the state of Sao Paulo, Southeastern Brazil (Anura, Leiuperidae). Zootaxa 2496:38-48

VALDUJO, P.H. 2011. Diversidade e distribuição de anfíbios no Cerrado: o papel dos fatores históricos e dos gradientes ambientais. Tese de Doutorado, Universidade de São Paulo, São Paulo.

VALDUJO, P.H., CAMACHO, A., RECODER, R.S., TEIXEIRA JUNIOR, M., GHELLERE, J.M.B., MOTT, T., NUNES, P.M.S., NOGUEIRA, C. \& RODRIGUES, M.T. 2011. Anfíbios da Estação Ecológica Serra Geral do Tocantins, região do Jalapão, Estados do Tocantins e Bahia. Biota Neotrop. 11(1): http://www.biotaneotropica.org.br/v11n1/pt/ abstract?article+bn03511012011 (último acesso em 22/07/2012).

VALDUJO, P.H., SILVANO, D.L., COLLI, G. \& MARTINS, M. 2012. Anuran species composition and distribution patterns in Brazilian Cerrado, a neotropical hotspot. S. A. J. H. 7(2):63-78. http://dx.doi. org/10.2994/057.007.0209

VASCONCELOS, T.S., RODRÍGUEZ, M.A. \& HAWKINS, B.A. 2011. Biogeographic distribution patterns of South American amphibians: a regionalization based on cluster analysis. Nat. Conserv. 9(1):67-72. http:// dx.doi.org/10.4322/natcon.2011.008

VASCONCELOS, T.S., SANTOS, T.G., HADDAD, C.F.B. \& ROSSAFERES, D.C. 2010. Climatic variables and altitude as predictors of anuran species richness and number of reproductive modes in Brazil. J. Trop. Ecol. 26:423-432. http://dx.doi.org/10.1017/S0266467410000167

VELOSO, H.P., RANGEL-FILHO, A.L.R. \& LIMA, J.C.A. 1991. Classificação da vegetação brasileira, adaptada a um sistema universal. IBGE, Rio de Janeiro.

VERDADE, V.K., VALDUJO, P.H., CARNAVAL, A.C., SCHIESARI, L., TOLEDO, L.F., MOTT, T., ANDRADE, G.V., ETEROVICK, P.C., MENIN, M., PIMENTA, B.V.S., NOGUEIRA, C., LISBOA, C.S., PAULA, C.D. \& SILVANO, D.L. 2012. A leap further: the Brazilian Amphibian Conservation Action Plan. Alytes 29(1-4):28-43.

WERNECK, F.P. \& COLLI, G.R. 2006. The lizard assemblage from Seasonally Dry Tropical Forest enclaves in the Cerrado biome, Brazil, and its association with the Pleistocenic Arc. J. Biogeogr. 33:1983-1992. http://dx.doi.org/10.1111/j.1365-2699.2006.01553.x

WERNECK, F.P. COSTA, G.C., COLLI, G.R., PRADO, D.E. \& SITES JUNIOR, J.W. 2011. Revisiting the historical distribution of Seasonally Dry Tropical Forests: new insights based on palaeodistribution modelling and palynological evidence. Global Ecol. Biogeogr. 20:272-288. http:// dx.doi.org/10.1111/j.1466-8238.2010.00596.x 
Araujo, C.O. et al.

Apêndice 1. Espécies de anuros registradas nos municípios do Alto e Médio Paranapanema, SP. As informações foram obtidas em coleções herpetológicas (CFBH, DZSJRP, MZUSP e ZUEC) e na literatura. A citação foi feita prioritariamente com base nos dados oriundos das coleções. Na ausência destes registros, foram considerados artigos científicos, livros, dissertações e teses (Bertoluci 2001', Bertoluci \& Rodrigues 2002², Jim 2002 ${ }^{3}$, Antunes $2007^{4}$, Moraes et al.

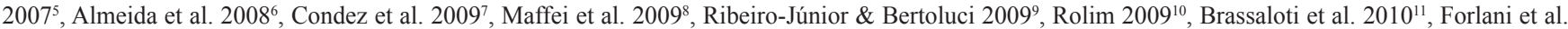
$2010^{12}$, Lopes $2010^{13}$, Maffei et al. $\left.2011 \mathrm{a}^{14}, \mathrm{~b}^{15}, \mathrm{c}^{16}\right)$.

Appendix 1. Anuran species recorded in the municipalities of Upper and Middle Paranapanema, São Paulo state, Brazil. The informations were obtained in herpetological collections (CFBH, DZSJRP, MZUSP and ZUEC) and literature. The citation was done primarily based on data from the collections. In the absence of these records, we considered scientific articles, books, dissertations and theses (Bertoluci 2001 ${ }^{1}$, Bertoluci \& Rodrigues $2002^{2}$, Jim $2002^{3}$, Antunes $2007^{4}$, Moraes et al. 20075, Almeida et al. 2008 ${ }^{6}$, Condez et al. 20097, Maffei et al. 2009 ${ }^{8}$, Ribeiro-Júnior \& Bertoluci 2009 , Rolim $2009^{10}$, Brassaloti et al. $2010^{11}$, Forlani et al. $2010^{12}$, Lopes $2010^{13}$, Maffei et al. $\left.2011 \mathrm{a}^{14}, \mathrm{~b}^{15}, \mathrm{c}^{16}\right)$.

\section{FAMÍLIA BRACHYCEPHALIDAE}

Brachycephalus hermogenesi

Registros: Alto Paranapanema: Piedade (CFBH 29653); Tapiraí ${ }^{7}$

Ischnocnema cf. hoehnei

Registros: Alto Paranapanema: Pilar do Sul (CFBH 8336).

Ischnocnema cf. spanios

Registros: Alto Paranapanema: Pilar do Sul (CFBH 16557); São Miguel Arcanjo (MZUSP 135942).

Ischnocnema guentheri

Registros: Alto Paranapanema: Apiaí (CFBH 25606); Guapiara (CFBH 13473); Piedade (CFBH 22262); Pilar do Sul (CFBH 16503); Ribeirão Branco (CFBH 2308); Ribeirão Grande (ZUEC 13746); São Miguel Arcanjo (MZUSP 136671); Tapiraí (CFBH 16550).

\section{Ischnocnema parva}

Registros: Alto Paranapanema: Apiaí (CFBH 25608); Capão Bonito (ZUEC 9212); Piedade ${ }^{7}$ Pilar do Sul (CFBH 7560); Ribeirão Grande (ZUEC 13752); São Miguel Arcanjo (MZUSP 135929); Tapiraí7.

\section{FAMÍLIA BUFONIDAE}

\section{Dendrophryniscus brevipollicatus}

Registros: Alto Paranapanema: Capão Bonito (ZUEC 9214); Piedade (CFBH 22285); Pilar do Sul (CFBH 8349); Ribeirão Grande'; São Miguel Arcanjo (MZUSP 136523); Tapiraí (CFBH 16538).

\section{Rhinella hoogmoedi}

Registros: Alto Paranapanema: Capão Bonito (MZUSP 69812); Ribeirão Grande'; São Miguel Arcanjo (ZUEC 15799).

\section{Rhinella icterica}

Registros: Alto e Médio Paranapanema: Apiaí (CFBH 25621); Botucatu³; Capão Bonito (ZUEC 9203); Guapiara (CFBH 13472); Piedade7; Pilar do Sul (CFBH 8604); Ribeirão Branco (CFBH 6904); Ribeirão Grande (ZUEC 13759); São Miguel Arcanjo (MZUSP 136614); Tapiraí ${ }^{7}$

\section{Rhinella ornata}

Registros: Alto e Médio Paranapanema: Águas de Santa Bárbara (MZUSP 151036); Angatuba e Guareí (ZUEC 18878); Apiaí (CFBH 25672); Borebi ${ }^{15}$; Botucatu (DZSJRP 6013); Buri (ZUEC 14581); Capão Bonito (MZUSP 136652); Gália (DZSJRP 13620); Garça (DZSJRP 13851); Guapiara (CFBH 13471); Lençóis Paulista ${ }^{14}$; Lupércio (DZSJRP 13878); Piedade (ZUEC 12091); Pilar do Sul (CFBH 16561); Piraju (MZUSP 132186); Piratininga (DZSJRP 13766); Pratânia ${ }^{10}$; Ribeirão Branco (CFBH 6902); Ribeirão Grande (ZUEC 13764); São Manuel $^{10}$; São Miguel Arcanjo (MZUSP 136643); Tapiraí7.

\section{Rhinella schneideri}

Registros: Alto e Médio Paranapanema: Águas de Santa Bárbara (MZUSP 151038); Angatuba e Guareí (ZUEC 20369); Assis (CFBH 18862); Borebi $^{15}$; Botucatu ${ }^{3}$; Buri (MZUSP 128536); Gália ${ }^{11}$; Garça (DZSJRP 1797); Lençóis Paulista ${ }^{14}$; Lupércio (DZSJRP 13891); Pratânia ${ }^{10}$; Ribeirão Grande (ZUEC 17212); São Manuel ${ }^{10}$.

\section{FAMÍLIA CENTROLENIDAE}

\section{Vitreorana eurygnatha}

Registros: Alto Paranapanema: Ribeirão Branco (CFBH 1854); Tapiraí (CFBH 16549).

\section{Vitreorana uranoscopa}

Registros: Alto e Médio Paranapanema: Angatuba e Guareí (ZUEC 18893); Botucatu (ZUEC 3679); Gália ${ }^{11}$; Lençóis Paulista ${ }^{14}$; Piedade (CFBH 22256); Pilar do Sul (CFBH 9318); São Miguel Arcanjo (MZUSP 136531); Tapiraí7.

\section{FAMÍLIA CERATOPHRYIDAE}

Ceratophrys aurita

Registros: Alto Paranapanema: Ribeirão Branco (CFBH 2320); Tapiraí (MZUSP 109270).

\section{FAMÍLIA CRAUGASTORIDAE}

\section{Haddadus binotatus}

Registros: Alto e Médio Paranapanema: Angatuba e Guareí (ZUEC 18841); Botucatu³; Buri (MZUSP 134852); Capão Bonito (MZUSP 135932); Gália (DZSJRP 13679); Garça (DZSJRP 13856); Guapiara (CFBH 14723); Piedade7; Pilar do Sul (CFBH 16499); Ribeirão Branco (CFBH 1778); Ribeirão Grande (ZUEC 18960); São Miguel Arcanjo (MZUSP 135930); Tapiraí7.

\section{FAMÍLIA CYCLORAMPHIDAE}

Registros: Alto Paranapanema: Guapiara (CFBH 14689); Piedade; Pilar do Sul (CFBH 3786); Ribeirão Grande (ZUEC 13751); São Miguel Arcanjo (MZUSP 138364); Tapiraí?. 
Apêndice 1. Continuação...

Appendix 1. Continued...

Cycloramphus eleutherodactylus

Registros: Alto Paranapanema: Ribeirão Grande (ZUEC 12087).

FAMÍLIA ODONTOPHRYNIDAE

Macrogenioglottus alipioi

Registros: Alto Paranapanema: Piedade7; Pilar do Sul (CFBH 6173); Ribeirão Branco (CFBH 6901); Ribeirão Grande (ZUEC 13762); São

Miguel Arcanjo (MZUSP 136625); Tapiraí7.

\section{Odontophrynus americanus}

Registros: Alto e Médio Paranapanema: Angatuba e Guareí (ZUEC 18866); Assis ; Borebi $^{15}$; Botucatu (ZUEC 19024); Buri (MZUSP 134818); Gália (DZSJRP 13684); Garça (DZSJRP 13861); Lençóis Paulista ${ }^{14}$; Lupércio (DZSJRP 13894); Piedade7; Ribeirão Grande ${ }^{1}$; Tapiraí ${ }^{7}$.

Odontophrynus cf. cultripes

Registros: Médio Paranapanema: Águas de Santa Bárbara (MZUSP 132970).

Proceratophrys boiei

Registros: Alto e Médio Paranapanema: Apiaí (CFBH 25669); Botucatu³; Buri (MZUSP 134809); Capão Bonito (ZUEC 9058); Guapiara (CFBH 13488); Piedade (CFBH 22265); Pilar do Sul (CFBH 16506); Ribeirão Branco (CFBH 4534); Ribeirão Grande (ZUEC 12376); São Miguel Arcanjo (MZUSP 136626); Tapiraí7.

Proceratophrys melanopogon

Registros: Alto Paranapanema: Pilar do Sul (CFBH 9312).

Proceratophrys moratoi

Registros: Alto e Médio Paranapanema: Avaré ${ }^{16}$; Botucatu (ZUEC 7031); Lençóis Paulista ${ }^{14}$.

FAMÍLIA HEMIPHRACTIDAE

Flectonotus fissilis

Registros: Alto Paranapanema: Piedade7; Pilar do Sul (CFBH 8614); Tapiraí (CFBH 10315).

Flectonotus ohausi

Registros: Alto Paranapanema: Apiaí (CFBH 25601); Pilar do Sul (CFBH 7552); Tapiraí (CFBH 16545).

Gastrotheca microdiscus

Registros: Alto Paranapanema: Capão Bonito (ZUEC 8995); Pilar do Sul (CFBH 7553); Ribeirão Grande ${ }^{1}$.

FAMÍLIA HYLIDAE

Aplastodiscus albosignatus

Registros: Alto Paranapanema: Guapiara (CFBH 13466); Piedade (CFBH 22246); Pilar do Sul (CFBH 9313); Ribeirão Branco (CFBH 9580); Ribeirão Grande ${ }^{1}$; São Miguel Arcanjo (MZUSP 136100).

Aplastodiscus callipygius

Registros: Alto Paranapanema: Apiaí (CFBH 25591).

Aplastodiscus cf. ehrhardti

Registros: Alto Paranapanema: Apiaí (CFBH 25647).

Aplastodiscus leucopygius

Registros: Alto Paranapanema: Piedade 7 ; Pilar do Sul14; Tapiraí7.

Aplastodiscus perviridis

Registros: Alto e Médio Paranapanema: Águas de Santa Bárbara (MZUSP 151012); Angatuba e Guareí (ZUEC 18812); Apiaí (CFBH 25592); Botucatu (DZSJRP 1854); Buri (MZUSP 134888); Guapiara (CFBH 14705); Itatinga (ZUEC 8386); Lençóis Paulista ${ }^{14}$; Piedade ${ }^{7}$; Pilar do Sul (CFBH 6204); Piraju (MZUSP 72380); Ribeirão Branco (CFBH 2371); Ribeirão Grande (ZUEC 13788); São Manuel ${ }^{10}$; Tapiraí7.

Bokermannohyla astartea

Registros: Alto Paranapanema: Pilar do Sul'; São Miguel Arcanjo (MZUSP 136541); Tapiraí (CFBH 15570).

Bokermannohyla circumdata

Registros: Alto Paranapanema: Apiaí (CFBH 25649); Buri (MZUSP 134892); Capão Bonito (MZUSP 136037); Guapiara (CFBH 13484); Piedade (CFBH 22247); Pilar do Sul (CFBH 15578); Ribeirão Grande (DZSJRP 8406); São Miguel Arcanjo (MZUSP 136030); Tapiraí (CFBH 15573).

\section{Bokermannohyla hylax}

Registros: Alto Paranapanema: Apiaí (CFBH 25593); Capão Bonito (MZUSP 136089); Piedade7; Pilar do Sul (CFBH 15580); Ribeirão Grande (DZSJRP 8404); São Miguel Arcanjo (MZUSP 136040); Tapiraí (CFBH 15568).

Bokermannohyla izecksohni

Registros: Médio Paranapanema: Botucatu (ZUEC 11543).

Bokermannohyla luctuosa

Registros: Alto Paranapanema: Ribeirão Branco (CFBH 30908).

Dendropsophus anceps

Registros: Alto e Médio Paranapanema: Angatuba e Guareí (ZUEC 18817); Borebi ${ }^{15}$; Buri (MZUSP 134907); Lençóis Paulista ${ }^{14}$. 
Araujo, C.O. et al.

Apêndice 1. Continuação...

Appendix 1. Continued..

\section{Dendropsophus elegans}

Registros: Alto Paranapanema: Apiaí (CFBH 25597); Buri (MZUSP 134878); Ribeirão Grande (DZSJRP L1861.1); São Miguel Arcanjo (MZUSP 135910).

\section{Dendropsophus elianeae}

Registros: Alto e Médio Paranapanema: Águas de Santa Bárbara (MZUSP 151058); Angatuba ${ }^{13}$; Assis (MZUSP 124224); Borebi ${ }^{15}$; Botucatu (ZUEC 12273); Buri (MZUSP 128496); Gália (DZSJRP 13687); Itapeva (MZUSP 60862); Lençóis Paulista ${ }^{14}$; Pratânia ${ }^{10}$.

\section{Dendropsophus giesleri}

Registros: Alto Paranapanema: Ribeirão Branco (ZUEC 6564); Ribeirão Grande' ; São Miguel Arcanjo (MZUSP 136064); Tapiraí (CFBH 15574).

\section{Dendropsophus jimi}

Registros: Alto e Médio Paranapanema: Águas de Santa Bárbara (MZUSP 151018); Angatuba e Guareí (ZUEC 18823); Borebi ${ }^{15}$; Botucatu (ZUEC 13467); Buri (MZUSP 134942); Itapeva (MZUSP 60866); Lençóis Paulista ${ }^{14}$; Pratânia ${ }^{10}$.

\section{Dendropsophus microps}

Registros: Alto e Médio Paranapanema: Apiaí (CFBH 25594); Avaré8; Botucatu; Capão Bonito (MZUSP 135941); Guapiara (CFBH 13476); Lençóis Paulista ${ }^{14}$; Piedade (CFBH 22250); Pilar do Sul (CFBH 9343); Ribeirão Branco (CFBH 16598); Ribeirão Grande (DZSJRP 8407); São Miguel Arcanjo (MZUSP 135935); Tapiraí (CFBH 15576).

\section{Dendropsophus minutus}

Registros: Alto e Médio Paranapanema: Águas de Santa Bárbara (MZUSP 151056); Alvinlândia e Gália (DZSJRP 9424); Angatuba e Guareí (ZUEC 18825); Apiaí (CFBH 25599); Assis (CFBH 20022); Borebi ${ }^{15}$; Botucatu (CFBH 22408); Buri (MZUSP 128519); Capão Bonito (CFBH 10678); Guapiara (CFBH 13462); Itatinga ${ }^{10}$; Lençóis Paulista (ZUEC 20225); Marília (CFBH 30474); Ourinhos (ZUEC 18952); Piedade (CFBH 22252); Pilar do Sul (CFBH 9316); Piraju (MZUSP 72391); Pratânia ${ }^{10}$; Ribeirão Branco (CFBH 16610); Ribeirão Grande (ZUEC 13756); São Manuel ${ }^{10}$; São Miguel Arcanjo (MZUSP 136077).

\section{Dendropsophus nanus}

Registros: Alto e Médio Paranapanema: Águas de Santa Bárbara (MZUSP 151098); Angatuba e Guareí (ZUEC 18827); Assis (CFBH 18857); Borebi ${ }^{15}$; Botucatu (CFBH 14617); Buri (MZUSP 134893); Gália (DZSJRP 13688); Guapiara (CFBH 14706); Itapetininga (CFBH 19353); Itatinga ${ }^{10}$; Lençóis Paulista ${ }^{14}$; Ourinhos (ZUEC 18951); Paranapanema (ZUEC 7155); Pratânia ${ }^{10}$; Ribeirão Branco (CFBH 11284); Ribeirão Grande (ZUEC 17795); São Manuel ${ }^{10}$.

\section{Dendropsophus sanborni}

Registros: Alto e Médio Paranapanema: Angatuba e Guareí (ZUEC 18832); Botucatu (CFBH 14621); Buri (MZUSP 134949); Capão Bonito (MZUSP 136673); Guapiara (CFBH 13464); Itatinga ${ }^{10}$; Lençóis Paulista14 ${ }^{14}$ Marília (CFBH 30471); Piedade7; Pilar do Sul (CFBH 9317); Pratânia ${ }^{10}$; Ribeirão Branco (CFBH 11283); Ribeirão Grande (ZUEC 13748); São Manuel ${ }^{10}$; Tapiraí7.

\section{Dendropsophus seniculus}

Registros: Alto Paranapanema: Apiaí (CFBH 25600); Capão Bonito (MZUSP 135944); Piedade (CFBH 22253); Ribeirão Branco (CFBH 2228); Ribeirão Grande (ZUEC 17225); São Miguel Arcanjo (MZUSP 135943).

\section{Dendropsophus werneri}

Registros: Alto Paranapanema: Ribeirão Grande ${ }^{2}$

\section{Hypsiboas albomarginatus}

Registros: Alto Paranapanema: Angatuba ${ }^{13}$; Piedade7 ; Ribeirão Branco (ZUEC 6569); São Miguel Arcanjo (MZUSP 136672); Tapiraí7.

\section{Hypsiboas albopunctatus}

Registros: Alto e Médio Paranapanema: Águas de Santa Bárbara (MZUSP 151031); Angatuba e Guareí (ZUEC 18844); Assis (CFBH 18840); Borebi $^{15}$; Botucatu (ZUEC 7575); Buri (MZUSP 134922); Gália ${ }^{11}$; Itatinga ${ }^{10}$; Lençóis Paulista ${ }^{14}$; Marília (CFBH 30470); Piedade (CFBH 22258); Pilar do Sul (CFBH 6215); Piraju (MZUSP 72381); Pratânia ${ }^{10}$; Ribeirão Branco (CFBH 6820); Ribeirão Grande (ZUEC 13777); São Manuel (DZSJRP 2508); São Miguel Arcanjo (MZUSP 136133); Tapiraí7.

\section{Hypsiboas bischoffi}

Registros: Alto Paranapanema: Buri (MZUSP 134910); Apiaí (CFBH 25655); Capão Bonito (ZUEC 8916); Guapiara (CFBH 13458); Piedade (CFBH 22260); Pilar do Sul (CFBH 6213); Ribeirão Branco (CFBH 11297); Ribeirão Grande (ZUEC 13765); São Miguel Arcanjo (MZUSP 136506); Tapiraí?

\section{Hypsiboas caingua}

Registros: Alto e Médio Paranapanema: Águas de Santa Bárbara (MZUSP 151010); Assis (CFBH 18691); Botucatu (CFBH 29525); Buri (MZUSP 128470); Gália ${ }^{11}$; Garça (DZSJRP 13864); Guapiara (CFBH 14697); Itatinga ${ }^{10}$; Lençóis Paulista ${ }^{14}$; Piedade ${ }^{7}$; Pilar do Sull Piraju $^{4}$ (MZUSP 72404); Pratânia ${ }^{10}$; Ribeirão Grande (ZUEC 13780); São Manuel ${ }^{10}$; Tapiraí ${ }^{7}$.

\section{Hypsiboas caipora}

Registros: Alto Paranapanema: Capão Bonito ${ }^{12}$; Pilar do Sul (CFBH 19231); São Miguel Arcanjo ${ }^{12}$.

Hypsiboas faber

Registros: Alto e Médio Paranapanema: Alvinlândia e Gália (DZSJRP 9419); Angatuba e Guareí (ZUEC 18845); Apiaí (CFBH 25602); Assis (ZUEC 10033); Borebi ${ }^{15}$; Botucatu (ZUEC 1226); Buri (MZUSP 134849); Garça (DZSJRP 1344); Guapiara (CFBH 13467); Itatinga ${ }^{10}$; Lençóis Paulista ${ }^{14}$; Piedade7; Pilar do Sul (CFBH 16562); Pratânia ${ }^{10}$; Ribeirão Branco (CFBH 8056); Ribeirão Grande (ZUEC 13772); São Manuel $^{10}$; São Miguel Arcanjo (MZUSP 136062); Tapiraí7. 
Apêndice 1. Continuação...

Appendix 1. Continued...

\section{Hypsiboas lundii}

Registros: Alto e Médio Paranapanema: Águas de Santa Bárbara (MZUSP 151077); Angatuba ${ }^{13}$; Assis (CFBH 20059); Borebi ${ }^{10}$; Botucatu (DZSJRP 5649); Gália (DZSJRP L2379.1); Lençóis Paulista ${ }^{14}$; Marília (DZSJRP 4072); São Manuel ${ }^{10}$.

Hypsiboas pardalis

Registros: Alto e Médio Paranapanema: Apiaí (CFBH 25603); Botucatu (ZUEC 1241); Piedade7; Pilar do Sul (CFBH 16563); Ribeirão

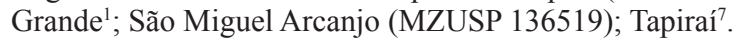

\section{Hypsiboas prasinus}

Registros: Alto e Médio Paranapanema: Angatuba e Guareí (ZUEC 18847); Apiaí (CFBH 25604); Botucatu (DZSJRP 2537); Capão Bonito (ZUEC 9207); Garça (DZSJRP 1337); Lençóis Paulista ${ }^{14}$; Piedade (CFBH 22261); Pilar do Sul (CFBH 6214); Piraju (MZUSP 72403); Ribeirão Branco (CFBH 30911); Ribeirão Grande (ZUEC 13754); São Miguel Arcanjo (CFBH 5613); Tapiraí? .

\section{Hypsiboas raniceps}

Registros: Médio Paranapanema: Ourinhos (ZUEC 18945).

Hypsiboas semilineatus

Registros: Alto Paranapanema: Piedade ${ }^{7}$; Tapiraí7.

Itapotihyla langsdorffii

Registros: Alto e Médio Paranapanema: Águas de Santa Bárbara (MZUSP 151063); Angatuba e Guareí (ZUEC 18851); Botucatu (CFBH 29531); Buri (MZUSP 134843); Gália ${ }^{11}$; Lençóis Paulista" ${ }^{14}$; Ribeirão Grande (ZUEC 18809).

\section{Phasmahyla cochranae}

Registros: Alto Paranapanema: Capão Bonito (MZUSP 16552); Ribeirão Grande (DZSJRP L1847).

\section{Phyllomedusa distincta}

Registros: Alto Paranapanema: Apiaí (CFBH 25617); Capão Bonito (MZUSP 136028); Pilar do Sul (CFBH 8334); Ribeirão Branco (CFBH 31060); Ribeirão Grande (ZUEC 13753); São Miguel Arcanjo (MZUSP 136027).

\section{Phyllomedusa tetraploidea}

Registros: Alto e Médio Paranapanema: Águas de Santa Bárbara (MZUSP 151074); Alvinlândia e Gália (DZSJRP 9420); Angatuba e Guareí (ZUEC 18867); Assis (CFBH 18829); Borebi ${ }^{15}$; Botucatu'; Buri (MZUSP 134838); Cerqueira César (MZUSP 12740); Garça (DZSJRP 1690); Lençóis Paulista ${ }^{14}$; Marília (CFBH 30477); Paranapanema (ZUEC 19083); Pilar do Sul (CFBH 8291); Pratânia ${ }^{10}$; Ribeirão Branco (CFBH 31051); Ribeirão Grande (DZSJRP 8100).

\section{Pseudis platensis}

Registros: Alto e Médio Paranapanema: Angatuba (CFBH 23159); Assis (MZUSP 124249); Botucatu³; Buri (MZUSP 134867); Itatinga (ZUEC 2371).

\section{Scinax alter}

Registros: Alto Paranapanema: São Miguel Arcanjo (MZUSP 136019).

\section{Scinax berthae}

Registros: Alto e Médio Paranapanema: Águas de Santa Bárbara (CFBH 22335); Apiaí (CFBH 25703); Borebi ${ }^{15}$; Botucatu (ZUEC 10673); Buri (MZUSP 134939); Lençóis Paulista ${ }^{14}$; São Manuel ${ }^{10}$.

\section{Scinax brieni}

Registros: Alto Paranapanema: Capão Bonito (MZUSP 136554); Pilar do Sul'4; Tapiraí (CFBH 15566).

\section{Scinax caldarum}

Registros: Alto Paranapanema: Ribeirão Branco (CFBH 6390).

Scinax cf. similis

Registros: Médio Paranapanema: Águas de Santa Bárbara (MZUSP 151090); Assis (CFBH 20052); Borebi ${ }^{15}$; Botucatu (DZSJRP 8051); Lençóis Paulista ${ }^{14}$; Pratânia ${ }^{10}$.

\section{Scinax crospedospilus}

Registros: Alto Paranapanema: Apiaí (CFBH 25622); Piedade (CFBH 22267); Pilar do Sul (CFBH 8932); Ribeirão Grande (ZUEC 17792); São Miguel Arcanjo (MZUSP 136009); Tapiraí?.

Scinax eurydice

Registros: Alto Paranapanema: Piedade (CFBH 22277).

Scinax fuscomarginatus

Registros: Alto e Médio Paranapanema: Águas de Santa Bárbara (MZUSP 151050); Angatuba e Guareí (ZUEC 18883); Assis (CFBH 20051); Borebi ${ }^{15}$; Botucatu (DZSJRP 149); Buri (MZUSP 134824); Gália (DZSJRP 13689); Itapetininga (CFBH 19354); Itapeva (MZUSP 60876); Itatinga ${ }^{10}$; Lençóis Paulista (ZUEC 20221); Ourinhos (ZUEC 18949); Piedade7; Pilar do Sul (CFBH 7629); Pratânia ${ }^{10}$; Ribeirão Branco (CFBH 4166); São Miguel Arcanjo (MZUSP 136562); Tapiraí7.

\section{Scinax fuscovarius}

Registros: Alto e Médio Paranapanema: Águas de Santa Bárbara (MZUSP 151029); Alvinlândia e Gália (DZSJRP 9423); Angatuba e Guareí (ZUEC 18888); Assis (CFBH 18841); Borebi ${ }^{15}$; Botucatu (DZSJRP 6074); Buri (ZUEC 14579); Capão Bonito (MZUSP 136022); Guapiara (CFBH 14699); Lençóis Paulista14; Marília (DZSJRP 2701); Ourinhos (CFBH 14072); Pilar do Sul (CFBH 7627); Pratânia ${ }^{10}$; Quatá (DZSJRP 2202); Rancharia (DZSJRP 2255); Ribeirão Branco (CFBH 30910); Ribeirão Grande (ZUEC 13785); São Manuel10; São Miguel Arcanjo (MZUSP 136026). 
Apêndice 1. Continuação...

Appendix 1. Continued...

\section{Scinax hayii}

Registros: Alto Paranapanema: Piedade (CFBH 22268); Ribeirão Grande ${ }^{1}$; São Miguel Arcanjo 5 .

Scinax hiemalis

Registros: Médio Paranapanema: Botucatu (CFBH 4544); Gália ${ }^{11}$; São Manuel ${ }^{10}$.

Scinax littoralis

Registros: Alto Paranapanema: Tapiraí (MZUSP 125393).

Scinax obtriangulatus

Registros: Alto Paranapanema: Ribeirão Grande ${ }^{1}$.

Scinax perereca

Registros: Alto e Médio Paranapanema: Apiaí (CFBH 24623); Gália" ${ }^{11}$; Guapiara (CFBH 13452); Piedade7; Pilar do Sul (CFBH 8350);

Ribeirão Branco (CFBH 30918); Ribeirão Grande (DZSJRP L1863.4); São Miguel Arcanjo (MZUSP 136116); Tapiraí (CFBH 16551).

Scinax perpusillus

Registros: Alto Paranapanema: Apiaí (CFBH 25674); Pilar do Sul (CFBH 7564); Ribeirão Grande'; São Miguel Arcanjo (MZUSP 136025);

Tapiraí (CFBH 16546).

Scinax rizibilis

Registros: Alto e Médio Paranapanema: Alvinlândia e Gália (DZSJRP 9427); Angatuba e Guareí (ZUEC 19201); Apiaí (CFBH 25624);

Capão Bonito (MZUSP 136612); Guapiara (CFBH 13455); Piedade (CFBH 22271); Pilar do Sul (CFBH 7628); Ribeirão Branco (CFBH

13647); Ribeirão Grande (ZUEC 13504); São Miguel Arcanjo (MZUSP 136611); Tapiraí7.

Scinax squalirostris

Registros: Alto e Médio Paranapanema: Águas de Santa Bárbara (MZUSP 151084); Angatuba e Guareí (ZUEC 18890); Borebi ${ }^{15}$; Botucatu (ZUEC 1245); Lençóis Paulista ${ }^{14}$.

Scinax $x$-signatus (aff. similis)

Registros: Médio Paranapanema: Ourinhos (ZUEC 18954).

Sphaenorhynchus caramaschii

Registros: Alto e Médio Paranapanema: Angatuba e Guareí (ZUEC 18891); Apiaí (CFBH 25627); Avaré6; Botucatu ${ }^{6}$; Lençóis Paulista (ZUEC 20234); Piedade (CFBH 22273); Pilar do Sul (CFBH 8289); Ribeirão Branco (ZUEC 15267); Ribeirão Grande (CFBH 15581); Tapiraí7.

Trachycephalus imitatrix

Registros: Alto Paranapanema: Capão Bonito (ZUEC 8993); Piedade (CFBH 22275); Ribeirão Grande (DZSJRP 9149).

Trachycephalus lepidus

Registros: Alto Paranapanema: Capão Bonito (MZUSP 136548).

Trachycephalus mesophaeus

Registros: Alto Paranapanema: Piedade (CFBH 22276).

Trachycephalus typhonius

Registros: Alto e Médio Paranapanema: Botucatu3; Marília (CFBH 30476); Ribeirão Branco (CFBH 2212).

\section{FAMÍLIA HYLODIDAE}

\section{Crossodactylus caramaschii}

Registros: Alto e Médio Paranapanema: Botucatu (ZUEC 11422); Capão Bonito (ZUEC 11991); Gália (DZSJRP 13691); Guapiara (CFBH 13468); Lupércio (DZSJRP 13918); Ourinhos (MZUSP 134086); Piedade7; Pilar do Sul (CFBH 4249); Ribeirão Branco (CFBH 1850); Ribeirão Grande (ZUEC 13783); São Miguel Arcanjo (MZUSP 136590); Tapiraí7.

Crossodactylus sp. (gr. gaudichaudii)

Registros: Médio Paranapanema: Rancharia (DZSJRP 4469).

Hylodes cf. asper

Registros: Alto Paranapanema: Guapiara (CFBH 13486); Ribeirão Grande' .

Hylodes cf. cardosoi

Registros: Alto Paranapanema: Capão Bonito (MZUSP 136571).

Hylodes heyeri

Registros: Alto Paranapanema: Capão Bonito (CFBH 10677).

Hylodes phyllodes

Registros: Alto Paranapanema: Piedade7; Tapiraí7.

Megaelosia cf. goeldii

Registros: Alto Paranapanema: Ribeirão Grande ${ }^{1}$.

\section{FAMÍLIA LEPTODACTYLIDAE}

\section{Adenomera cf. bokermanni}

Registros: Alto e Médio Paranapanema: Botucatu (ZUEC 3904); Guapiara (CFBH 13478); Lençóis Paulista ${ }^{14}$; Ribeirão Branco (CFBH 2306); Ribeirão Grande (ZUEC 13749). 
Apêndice 1. Continuação...

Appendix 1. Continued...

\section{Adenomera cf. marmorata}

Registros: Alto e Médio Paranapanema: Apiaí (CFBH 25662); Botucatu (ZUEC 2503); Buri (MZUSP 134921); Capão Bonito (ZUEC 11978);

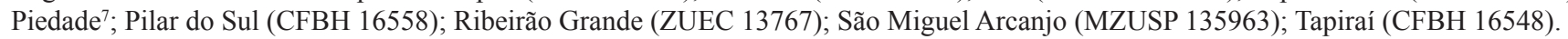

Adenomera sp.

Registros: Alto Paranapanema: Angatuba e Guareí (ZUEC 18864).

Eupemphix nattereri

Registros: Alto e Médio Paranapanema: Águas de Santa Bárbara (MZUSP 151070); Angatuba e Guareí (ZUEC 18836); Assis (CFBH 20018); Borebi ${ }^{15}$; Botucatu (DZSJRP 9068); Gália (DZSJRP 13694); Lençóis Paulista ${ }^{14}$; Ourinhos (ZUEC 18948); Piraju (MZUSP 132185); Piratininga (DZSJRP 13773); Pratânia ${ }^{10}$.

\section{Leptodactylus chaquensis}

Registros: Médio Paranapanema: Gália ${ }^{11}$.

\section{Leptodactylus flavopictus}

Registros: Alto Paranapanema: Ribeirão Branco (CFBH 4725); Ribeirão Grande'; São Miguel Arcanjo (MZUSP 136665).

Leptodactylus furnarius

Registros: Alto e Médio Paranapanema: Águas de Santa Bárbara (MZUSP 151088); Angatuba ${ }^{13}$; Assis (CFBH 20055); Botucatu (ZUEC 4078); Gália (DZSJRP 13721); Lençóis Paulista ${ }^{14}$.

\section{Leptodactylus fuscus}

Registros: Alto e Médio Paranapanema: Águas de Santa Bárbara (MZUSP 151004); Angatuba e Guareí (ZUEC 18856); Assis (CFBH 20054); Borebi ${ }^{15}$; Botucatu (DZSJRP 9069); Buri (MZUSP 134858); Capão Bonito (ZUEC 8918); Gália ${ }^{11}$; Lençóis Paulista ${ }^{14}$; Marília (CFBH 30475); Ourinhos (ZUEC 18946); Piedade7; Pilar do Sul (CFBH 9340); Piratininga (DZSJRP 13780); Pratânia ${ }^{10}$; Ribeirão Branco (CFBH 2327); Ribeirão Grande'; São Manuel ${ }^{10}$; São Miguel Arcanjo (MZUSP 135970); Tapiraí?.

Leptodactylus jolyi

Registros: Alto e Médio Paranapanema: Águas de Santa Bárbara (MZUSP 151034); Botucatu ${ }^{10}$; Piedade (CFBH 22289).

\section{Leptodactylus labyrinthicus}

Registros: Alto e Médio Paranapanema: Águas de Santa Bárbara (MZUSP 151096); Angatuba (CFBH 23157); Assis (CFBH 20049); Borebi15; Botucatu $^{3}$; Buri (MZUSP 134890); Gália ${ }^{11}$; Lençóis Paulista ${ }^{14}$; Lupércio (DZSJRP 13902); Pratânia ${ }^{10}$.

\section{Leptodactylus latrans}

Registros: Alto e Médio Paranapanema: Águas de Santa Bárbara (MZUSP 151039); Angatuba e Guareí (ZUEC 18858); Apiaí (CFBH 25613); Assis (CFBH 20057); Borebi ${ }^{15}$; Botucatu (ZUEC 1235); Buri (MZUSP 134833); Gália ${ }^{11}$; Guapiara (CFBH 13477); Itatinga ${ }^{10}$; Lençóis Paulista ${ }^{14}$; Lupércio (DZSJRP 13916); Piedade7; Pilar do Sul (CFBH 8352); Ribeirão Branco (CFBH 6900); Ribeirão Grande (ZUEC 13773); São Manuel ${ }^{10}$; São Miguel Arcanjo (MZUSP 136668); Tapiraí7.

\section{Leptodactylus mystaceus}

Registros: Alto e Médio Paranapanema: Águas de Santa Bárbara (MZUSP 151033); Angatuba (CFBH 23146); Borebi ${ }^{15}$; Botucatu³ ${ }^{3}$ Gália (DZSJRP 13722); Lençóis Paulista ${ }^{14}$; Lupércio (DZSJRP 13903); Piratininga (DZSJRP 13781); Pratânia ${ }^{10}$.

\section{Leptodactylus mystacinus}

Registros: Alto e Médio Paranapanema: Águas de Santa Bárbara (MZUSP 151066); Angatuba e Guareí (ZUEC 18859); Assis (CFBH 20060); Borebi ${ }^{15}$; Botucatu (DZSJRP 9065); Buri (MZUSP 134834); Gália (DZSJRP 13729); Garça (DZSJRP 13871); Itapeva (MZUSP 138177); Itararé (MZUSP 138161); Lençóis Paulista ${ }^{14}$; Marília (DZSJRP 2696); Ourinhos (DZSJRP 11632); Piedade ${ }^{7}$; Piratininga (DZSJRP 13782); Pratânia $^{10}$; Ribeirão Branco (CFBH 2499); São Manuel ${ }^{10}$; Tapiraí7.

\section{Leptodactylus notoaktites}

Registros: Alto Paranapanema: Apiaí (CFBH 25611); Capão Bonito (ZUEC 8525); Ribeirão Branco (CFBH 11282); Ribeirão Grande (ZUEC 13778); Tapiraí (MZUSP 125405).

\section{Leptodactylus podicipinus}

Registros: Alto e Médio Paranapanema: Angatuba e Guareí (ZUEC 18863); Assis (CFBH 20056); Botucatu (ZUEC 2887); Chavantes (DZSJRP 10819); Gália ${ }^{11}$; Lençóis Paulista" ${ }^{14}$; Marília (DZSJRP 2697); Quatá (DZSJRP 1976); São Manuel (DZSJRP 2542).

\section{Paratelmatobius cf. gaigeae}

Registros: Alto Paranapanema: Ribeirão Grande ${ }^{1}$.

Paratelmatobius sp. (aff. cardosoi)

Registros: Alto Paranapanema: Apiaí (CFBH 25616); Capão Bonito (MZUSP 135993); Piedade7; São Miguel Arcanjo (MZUSP 135979); Tapiraí (CFBH 16535).

\section{Physalaemus centralis}

Registros: Alto e Médio Paranapanema: Águas de Santa Bárbara (MZUSP 151079); Angatuba (CFBH 23149); Assis (MZUSP 124228); Borebi ${ }^{15}$; Botucatu (ZUEC 1237); Lençóis Paulista ${ }^{14}$; Pratânia ${ }^{10}$. 
Araujo, C.O. et al.

Apêndice 1. Continuação...

Appendix 1. Continued..

\section{Physalaemus cuvieri}

Registros: Alto e Médio Paranapanema: Águas de Santa Bárbara (MZUSP 151014); Angatuba e Guareí (ZUEC 18870); Apiaí (CFBH 25619);

Assis (CFBH 20047); Borebi ${ }^{15}$; Botucatu (DZSJRP 6019); Buri (MZUSP 134964); Capão Bonito (ZUEC 11981); Gália (DZSJRP 13695);

Garça (DZSJRP 13865); Guapiara (CFBH 13487); Itapetininga (MZUSP 60968); Itapeva (MZUSP 60868); Itararé (MZUSP 138190); Itatinga $^{10}$; Lençóis Paulista ${ }^{14}$; Lupércio (DZSJRP 13899); Marília (DZSJRP 1852); Ourinhos (CFBH 14070); Paranapanema (CFBH 10675); Piedade (CFBH 22263); Pilar do Sul (CFBH 7561); Piraju (MZUSP 72470); Piratininga (DZSJRP 13777); Pratânia ${ }^{10}$; Ribeirão Branco (CFBH 2357); Ribeirão Grande (ZUEC 13760); São Manuel ${ }^{10}$; São Miguel Arcanjo (MZUSP 135969); Tapiraí7.

\section{Physalaemus lateristriga}

Registros: Alto Paranapanema: Capão Bonito (ZUEC 11992); Ribeirão Branco (ZUEC 6579); Ribeirão Grande (ZUEC 17249).

\section{Physalaemus marmoratus}

Registros: Alto e Médio Paranapanema: Águas de Santa Bárbara (MZUSP 151062); Angatuba (CFBH 23139); Assis (CFBH 20030); Borebi ${ }^{15}$; Botucatu (ZUEC 8324); Buri (MZUSP 134862); Gália (DZSJRP 13718); Lençóis Paulista ${ }^{14}$; Ourinhos (ZUEC 18950); São Manuel (ZUEC 2335).

\section{Physalaemus olfersii}

Registros: Alto e Médio Paranapanema: Angatuba e Guareí (ZUEC 18876); Apiaí (CFBH 25620); Botucatu (ZUEC 2176); Buri (MZUSP 134906); Capão Bonito (ZUEC 9239); Gália (DZSJRP 13699); Itatinga ${ }^{10}$; Piedade (CFBH 22264); Pilar do Sul (CFBH 6106); Ribeirão Branco (CFBH 16587); Ribeirão Grande (ZUEC 18956); São Miguel Arcanjo (MZUSP 136101); Tapiraí (CFBH 16547).

\section{Physalaemus spiniger}

Registros: Alto Paranapanema: Apiaí (CFBH 25668).

Pseudopaludicola cf. falcipes

Registros: Médio Paranapanema: Botucatu (ZUEC 1263).

Pseudopaludicola cf. murundu

Registros: Médio Paranapanema: Águas de Santa Bárbara (MZUSP 151097).

Pseudopaludicola cf. mystacalis

Registros: Alto e Médio Paranapanema: Assis9; Botucatu (MZUSP 14480); Buri (MZUSP 134955).

Pseudopaludicola cf. saltica

Registros: Médio Paranapanema: Botucatu (ZUEC 1271).

\section{FAMÍLIA MICROHYLIDAE}

\section{Chiasmocleis albopunctata}

Registros: Alto e Médio Paranapanema: Águas de Santa Bárbara (MZUSP 151024); Angatuba e Guareí (ZUEC 18815); Assis (CFBH 20032); Borebi ${ }^{15}$; Botucatu (ZUEC 3785); Buri (MZUSP 134882); Gáliaa ${ }^{11}$; Garça (DZSJRP 13875); Lençóis Paulista ${ }^{14}$; Piratininga (DZSJRP 13783); Pratânia ${ }^{10}$.

\section{Chiasmocleis leucosticta}

Registros: Alto Paranapanema: Piedade (CFBH 22248); Pilar do Sul (CFBH 4250); Ribeirão Branco (CFBH 9149); São Miguel Arcanjo (MZUSP 136050); Tapiraí?

\section{Elachistocleis bicolor}

Registros: Alto e Médio Paranapanema: Águas de Santa Bárbara (MZUSP 151013); Alvinlândia e Gália (DZSJRP 9425); Angatuba (CFBH 23163); Assis (CFBH 20035); Buri (MZUSP 134923); Chavantes (DZSJRP 10821); Ourinhos (DZSJRP 11642); Paranapanema (CFBH 10676).

Elachistocleis cesarii

Registros: Alto e Médio Paranapanema: Águas de Santa Bárbara (MZUSP 151041); Angatuba e Guareí (ZUEC 18835); Borebi15; Botucatu³; Buri (MZUSP 134926); Gália ${ }^{11}$; Lençóis Paulista ${ }^{14}$; Pratânia ${ }^{10}$; São Manuel ${ }^{10}$.

Myersiella microps

Registros: Alto Paranapanema: Piedade7 ; Pilar do Sul (CFBH 6111); São Miguel Arcanjo (MZUSP 136674); Tapiraí7. 